\title{
Influence of calcium concentrations on the metabolic profile of dairy goats during the
}

\section{transitional period}

Influência das concentrações de cálcio no perfil metabólico de cabras leiteiras durante o período de transição

Influencia de las concentraciones de calcio en el perfil metabólico de las cabras lecheras durante el período de transición

Jobson Filipe de Paula Cajueiro ORCID: https://orcid.org/0000-0003-3228-2383 Universidade Federal Rural de Pernambuco, Brazil E-mail: jobson.filipe@gmail.com

Rodolfo José Cavalcanti Souto ORCID: https://orcid.org/0000-0003-3380-5596 Universidade Federal Rural de Pernambuco, Brazil E-mail: rodolfojcsouto@gmail.com

Elizabeth Hortêncio de Melo

ORCID: https://orcid.org/0000-0001-7406-1445 Universidade Federal Rural de Pernambuco, Brazil E-mail: elishmelo@hotmail.com

Cleyton Charles Dantas Carvalho

ORCID: https://orcid.org/0000-0002-2576-9962 Universidade Federal do Piauí, Brazil E-mail: cleytoncarvalho@gmail.com

Rafael José da Silva

ORCID: https://orcid.org/0000-0003-2364-4418

Universidade Federal de Minas Gerais, Brazil E-mail: rafaeljs15@hotmail.com

Pierre Castro Soares

ORCID https://orcid.org/0000-0002-5680-3940 Universidade Federal Rural de Pernambuco, Brazil E-mail: pcastro.pe@gmail.com

Carla Lopes de Mendonça

ORCID https://orcid.org/0000-0002-4985-4985 Universidade Federal Rural de Pernambuco, Brazil E-mail: carla.mendonca@ufrpe.br

José Augusto Bastos Afonso

ORCID https://orcid.org/0000-0002-6558-644X Universidade Federal Rural de Pernambuco, Brazil E-mail: afonsojab@gmail.br

\begin{abstract}
In order to differentiate groups of hypocalcemic (G1) and normocalcemic animals (G2) and infer the influence of subclinical hypocalcemia on metabolic profiles, this study determined calcium concentrations during the transitional period in 35 dairy goats healthy, pregnant, primiparous or multiparous, crossbreed or pure-bred dairy goats producing average $3 \mathrm{~kg} /$ day/goat. Therefore, blood samples were collected before (30,20 and 10 days before parturition), on the day of delivery and after parturition $(10,20,30,40,50$ and 60 days postpartum). The variables measured were glucose, nonesterified fatty acids, $\beta$-hydroxybutyrate, cholesterol, triglycerides, amylase, total protein, albumin, urea, creatinine, aspartate aminotransferase, gamma glutamyl transferase, creatine kinase, total calcium, phosphorus, magnesium, chlorides, cortisol and insulin, as well as ionized calcium $\left(\mathrm{Ca}^{++}\right)$, sodium and potassium. Goats were considered to have subclinical hypocalcemia if $\mathrm{Ca}^{++} \leq 0.72 \mathrm{mmol} / \mathrm{L}$. The data were analyzed by ANOVA. In subclinically hypocalcemic goats, serum concentrations of $\mathrm{Ca}^{++}$decreased earlier (10dbp) than in normocalcemic goats (parturition) and remained lower throughout the transitional period $(\mathrm{p}=0,004)$. Among the measured variables, $\mathrm{Ca}^{++}$showed greater influence on the NEFA, glucose, insulin and total calcium but also influenced the protein profile. Lower food intake by goats with subclinical hypocalcemia was one of the main factors interfering with the metabolic profile and likely the productivity of these animals. Studies should be conducted to measure the effects of subclinical disease on the production rates and on the emergence of other transitional period diseases.
\end{abstract}

Keywords: Biochemical markers; Hypocalcemia; Metabolic profile; Small ruminants; Transitional period. 


\begin{abstract}
Resumo
Com o objetivo de diferenciar grupos de animais hipocalcêmicos (G1) e normocalcêmicos (G2) e inferir a influência da hipocalcemia subclínica nos perfis metabólicos, este estudo determinou as concentrações de cálcio durante o período de transição em 35 cabras leiteiras saudáveis, prenhas, primíparas ou multíparas, mestiças ou puras, com produção média de $3 \mathrm{~kg} / \mathrm{dia} /$ cabra. Amostras de sangue foram coletadas antes (30, 20 e 10 dias antes do parto), no dia do parto e após o parto $(10,20,30,40,50$ e 60 dias pós-parto). As variáveis medidas foram glicose, ácidos graxos não esterificados, $\beta$-hidroxibutirato, colesterol, triglicerídeos, amilase, proteína total, albumina, ureia, creatinina, aspartato aminotransferase, gama glutamil transferase, creatina quinase, cálcio total, fósforo, magnésio, cloretos, cortisol e insulina, além de cálcio ionizado $\left(\mathrm{Ca}^{++}\right)$, sódio e potássio. As cabras foram consideradas com hipocalcemia subclínica se $\mathrm{Ca}^{++} \leq 0,72 \mathrm{mmol} / \mathrm{L}$. Os dados foram analisados por ANOVA. Em cabras subclinicamente hipocalcêmicas, as concentrações séricas de $\mathrm{Ca}^{++}$diminuíram mais cedo (10dbp) do que em cabras normocalcêmicas (parto) e permaneceram mais baixas durante o período de transição $(\mathrm{p}=0,004)$. Dentre as variáveis medidas, o $\mathrm{Ca}^{++}$apresentou maior influência sobre os AGNE, glicose, insulina e cálcio total, mas também influenciou o perfil proteico. O menor consumo de ração por caprinos com hipocalcemia subclínica foi um dos principais fatores que interferiu no perfil metabólico e provável na produtividade desses animais. Estudos devem ser realizados para medir os efeitos da doença subclínica nas taxas de produção e no surgimento de outras doenças do período de transição.
\end{abstract}

Palavras-chave: Hipocalcemia; Marcadores bioquímicos; Pequenos ruminantes; Perfil metabólico; Período de transição.

\title{
Resumen
}

Para diferenciar grupos de animales hipocalcémicos (G1) y normocalcémicos (G2) e inferir la influencia de la hipocalcemia subclínica en los perfiles metabólicos, este estudio determinó las concentraciones de calcio durante el período de transición en 35 cabras sanas, gestantes, primíparas o lecheras multíparas, mestizas o puro, con una producción media de $3 \mathrm{~kg} /$ día/cabra. Las muestras de sangre se recolectaron antes (30, 20 y 10 días antes del parto), el día del parto y después del parto $(10,20,30,40,50$ y 60 días después del parto). Las variables medidas fueron glucosa, ácidos grasos no esterificados, $\beta$-hidroxibutirato, colesterol, triglicéridos, amilasa, proteína total, albúmina, urea, creatinina, aspartato aminotransferasa, gamma glutamil transferasa, creatina quinasa, calcio total, fósforo, magnesio, cloruros, cortisol e insulina, además de calcio ionizado $\left(\mathrm{Ca}^{++}\right)$, sodio y potasio. Se consideró que las cabras tenían hipocalcemia subclínica si $\mathrm{Ca}^{++} \leq 0,72 \mathrm{mmol} / \mathrm{L}$. Los datos fueron analizados por ANOVA. En cabras subclínicamente hipocalcémicas, las concentraciones séricas de $\mathrm{Ca}^{++}$disminuyeron antes(10dbp) que en las cabras normocalcémicas(paridas) y permanecieron más bajas durante el período de transición $(\mathrm{p}=0,004)$. Entre las variables medidas, $\mathrm{Ca}^{++}$tuvo la mayor influencia sobre NEFA, glucosa, insulina y calcio total, pero también influyó en el perfil proteico. El menor consumo de alimento de las cabras con hipocalcemia subclínica fue uno de los principales factores que interfirió en el perfil metabólico y probablemente en la productividad de estos animales. Deben realizarse estudios para medir los efectos de las enfermedades subclínicas en las tasas de producción y la aparición de otras enfermedades del período de transición.

Palabras clave: Hipocalcemia; Marcadores bioquímicos; Pequeños rumiantes; Perfil metabólico; Periodo de transición.

\section{Introduction}

The transitional period is considered the most critical for female ruminants because the passage from a pregnant nonlactating state to a postpregnancy lactating state leads to stress that is caused by large and abrupt changes in metabolism, anatomy and physiology (Grummer, 1995; Rabelo et al., 2005). This change, in association with the growing demand for productivity and individual efficiency, increases the frequency of digestive and metabolic disorders during this period. Among these disorders, ruminal acidosis, pregnancy toxemia and hypocalcemia stand out, all of which may occur in clinical or subclinical form (Moreno-Rojas et al., 1994; Goff \& Horst, 1997; Brozos et al., 2011). Hypocalcemia is a nonfebrile and progressive neuromuscular dysfunction that more frequently affects high-producing dairy cows but also occurs in small ruminants. It causes flaccid paralysis, circulatory collapse and sensory depression (Smith \& Sherman, 2009; Constable et al., 2017). This disorder occurs because of increased ionized calcium $\left(\mathrm{Ca}^{++}\right)$movement out of the blood plasma, which is not balanced by increasing absorption of this element in the intestine or by increasing bone resorption (Kimberling, 1988).

Unlike the form that occurs in dairy cows, which usually shows clinical signs within 24 to 48 hours of the second or third parity (Goff \& Horst, 1997), the disease in small ruminants can occur a few weeks before to 8 weeks after parturition 
(Darrell et al., 2005). Because of its association with the postpartum period and the beginning of the lactation period, the disease is also known as milk fever or puerperal paralysis (Ortolani, 1995).

The highest incidence of clinical and subclinical hypocalcemia occurs in the transitional period when there is a greater demand for this mineral for fetal skeleton development (Kimberling, 1988; Moreno-Rojas et al., 1994), colostrum formation and subsequently milk production (Goff \& Horst, 1997; Liesegang \& Risteli, 2005, Santos, 2011). Due to this high metabolic demand for calcium in peripartum periods, the homeostatic mechanisms that control the serum concentrations of this element often fail, leading cows (Reinhardt et al., 2011), ewes (Kimberling, 1988) and goats (Bruére \& West, 1993, Smith \& Sherman, 2009) to develop some degree of hypocalcemia around the time of parturition. Numerous epidemiological studies have clearly shown that

cows with a metabolic disorder, such as hypocalcemia, have a much higher risk of developing other diseases, such as ketosis, retained fetal membranes, mastitis and displaced abomasum, than cows that do not have this metabolic disorder (Goff, 2006; Goff, 2008).

Because of the importance of $\mathrm{Ca}^{++}$in many biological processes, the influence of hypocalcemia on animal production and the social connotation of the goat in northeastern Brazil, this study aimed to determine calcium concentrations during the transitional period in dairy goats, thereby differentiating groups of hypocalcemic and normocalcemic animals and inferring the influence of subclinical hypocalcemia on energy, protein, enzyme, hormone and mineral profiles.

\section{Materials and Methods}

Thirty-five healthy, pregnant, primiparous or multiparous, crossbred or pure-bred dairy goats—Saanen, Alpine Brown, American Alpine and Toggenburg were used. The goats had a mean weight of $60 \mathrm{~kg}$ (about 3,3 kg/day/goat of milk yield) and were maintained on three farms located in a semi-arid region of northeastern Brazil. The animals were bred intensively, and the diet did not vary among the properties; however, all goats received sugarcane bagasse (Saccharum sp., 360g kg/day of MS), palm (Opuntia tuna (L.) Mill, 360g kg/day of MS), corn bran (593g kg/day of MS), wheat bran (209g kg/day of MS), cottonseed meal (349g kg/day of MS), soybean meal (349g kg/day of MS), mineral salt ${ }^{1}$ (ad libitum) and water (ad libitum). Therefore, disregarding the amount and the insignificant nutritional value of the sugarcane bagasse, the diet provided contained 2.41 Mcal of ME/kg of MS (high energy) and $15.64 \% \mathrm{CP}$.

Blood samples were collected by jugular venipuncture into tubes with and without anticoagulant (sodium fluoride/oxalate) to obtain serum and plasma, respectively. The tubes were centrifuged ${ }^{2}$ at 3500 rpm for 10 minutes, and the serum and plasma were stored at $-80^{\circ} \mathrm{C}^{3}$ in $1.5 \mathrm{ml}$ microtubes for further laboratory processing. Collections of samples were performed as follows: at 30, 20 and 10 days before parturition (dbp); at the time of parturition; and at 10, 20, 30, 40, 50 and 60 days postpartum (dpp) (moments 1 to 10$)$.

The serum activities of enzymes aspartate aminotransferase (AST) ${ }^{4}$, gamma glutamyl transferase $(\mathrm{GGT})^{4}$, amylase ${ }^{4}$ and creatine kinase $(\mathrm{CK})^{4}$ as well as the concentration of total protein ${ }^{4}$, albumin ${ }^{4}$, urea $^{4}$, creatinine ${ }^{4}$, cholesterol ${ }^{4}$, triglycerides ${ }^{4}$, calcium $^{4}$, phosphorus $(\mathrm{P})^{4}$, magnesium ${ }^{4}$ and chlorides ${ }^{4}$, were evaluated using commercial kits (Labtest Diagnóstica S.A., Minas Gerais, Brazil), and the processing was performed in a semiautomatic analyzer (Labtest Diagnóstica S.A., Minas Gerais, Brazil). The ionized calcium $\left(\mathrm{Ca}^{++}\right)^{5}$, sodium $\left(\mathrm{Na}^{+}\right)^{5}$ and potassium $\left(\mathrm{K}^{+}\right)^{5}$ levels were determined with an electrolyte analyzer (Roche Diagnostics Ltd, São Paulo, Brazil).

\footnotetext{
${ }^{1}$ Ovinofós, DSM Produtos Nutricionais Brasil S.A., Av Alberto Cocozza 3000, Goiania, Mairinque, SP, Brazil

${ }^{2}$ Centrifuga Fanem Ltda Baby I, Mod. 206, Av. General Ataliba Leonel 1790, São Paulo, SP, Brazil.

${ }^{3}$ Ultralow freezer NuAire Inc., 2100 Fernbrook Lane N. Plymouth, MN 55447, USA.

${ }^{4}$ Labtest Diagnóstica S.A., Av. Paulo Ferreira da Costa 600, Lagoa Santa, 33400-000, Minas Gerais, MG, Brazil.

${ }^{5}$ Analizador de Íons, Roche Sistemas de Diagnósticos, Lda. Av. Eng. Billings, 1729, Jaguaré -São Paulo, 05321 - 900.
} 
The nonesterified fatty acids (NEFA) ${ }^{6}$ and $\beta$-hydroxybutyrate $(\mathrm{BHB})^{6}$ were determined with the semi-automatic analyzer using commercial kits (Randox Laboratories Ltd., Crumlin, United Kingdom). For the determination of $\operatorname{cortisol}^{7}$ and insulin ${ }^{7}$ hormone, the electrochemiluminescence method was performed with commercial kits (Cobas-Roche Diagnostics Ltd, São Paulo, Brazil). Plasma glucose determinations were performed according to the manufacturer's recommendations and processed on the semiautomatic analyzer ${ }^{4}$ (Labtest Diagnóstica S.A., Minas Gerais, Brazil).

A cutoff point of $\mathrm{Ca}^{++}$for subclinical hypocalcemia determination in dairy goats was based on the results obtained by Simplício et al., (2009), who investigated the $\mathrm{Ca}^{++}$concentrations in dairy goats and found a normal value of $0.83 \pm$ $0.1 \mathrm{mmol} / \mathrm{L}$.

Thus, goats were considered as having subclinical hypocalcemia when the serum concentration of $\mathrm{Ca}^{++}$in at least one of those transitional periods was lower than the lowest limit given by the standard deviation found by Simplício et al, (2009) $\left(\mathrm{Ca}^{++}<0.73 \mathrm{mmol} / \mathrm{L}\right)$ and when the goat had shown no clinical symptoms of "milk fever." The 35 goats were divided into two groups: hypocalcemic $(\mathrm{G} 1=15)$ and normocalcemic $(\mathrm{G} 2=20)$.

This is an analytical cohort study according to Thrusfield et al. (2018). The results for the variables in each group were statistically analyzed over the ten experimental time points, comparing the time points to each other, and the studied variables were submitted to ANOVA. The F statistics were considered significant when $\mathrm{P}<0.05$. The contrasts between means were performed by Tukey's test. The Mann-Whitney nonparametric test for independent samples (group effect) and the Friedman test for dependent samples (time effect) were used for the variables, using the median as a measure of central tendency. The $\chi^{2}$ test was used to calculate the msd for $\alpha=0.05$. The Pearson correlation coefficients were also used to assess the relationships between pairs of variables (Curi 1997). The computer program Sigma Stat 3.1 (Systat Software, San Jose, California, U.S.A.) was used for these analyses.

Ethics Committee: The project obtained a favorable ruling from the Animal Use Ethics Committee (CEUA Comissão de Ética no Uso de Animais) of the Universidade Federal Rural de Pernambuco (UFRPE), which granted the license number 047/2013 CEPE/UFRPE according to the rules of Brazilian College of Animal Experimentation (COBEA Colégio Brasileiro de Experimentação Animal) and the National Institutes of Health Guide for Laboratory Animals Care and Use.

\section{Results}

Of 15 goats with subclinical hypocalcemia (G1), 46.66\% (n=7) were affected by subclinical pregnancy toxemia. However, in the normocalcemic animals (G2), only 5\% $(\mathrm{n}=1)$ had this type of disorder. No clinical changes were observed during the study period in either group. At different times, a significant decrease was observed $(\mathrm{P}<0.001)$ in the ionized calcium values at parturition $(0.81 \mathrm{mmol} / \mathrm{L})$ in $\mathrm{G} 1$ compared to the initial time point $(30 \mathrm{dbp})$, whereas this happened at 10 dap $(0.92 \mathrm{mmol} / \mathrm{L})$ in $\mathrm{G} 2$. After these periods, an increase was observed in the concentrations of this variable, but these concentrations did not return to the indexes obtained initially. In a comparison of the groups, the G1 showed the lowest concentrations, and differences $(\mathrm{P}<0.04)$ were observed between the $10 \mathrm{dbp}$ to the postpartum period, except at 40 dap and 60 dap (Table 1; Figure 1).

\footnotetext{
${ }^{6}$ Randox Laboratories Ltd, Ardmore, Diamond Road, Crumlin, Co. Antrim, United Kingdom BT 29 4QY, UK.

${ }^{7}$ Cobas e 411 - Roche Sistemas de Diagnósticos, Lda. Av. Eng. Billings, 1729, Jaguaré - São Paulo, 05321 - 900.
} 
Figure 1: Mean values of $\mathrm{Ca}^{++}$and total calcium in hypocalcemic and normocalcemic dairy goats during the transitional period.

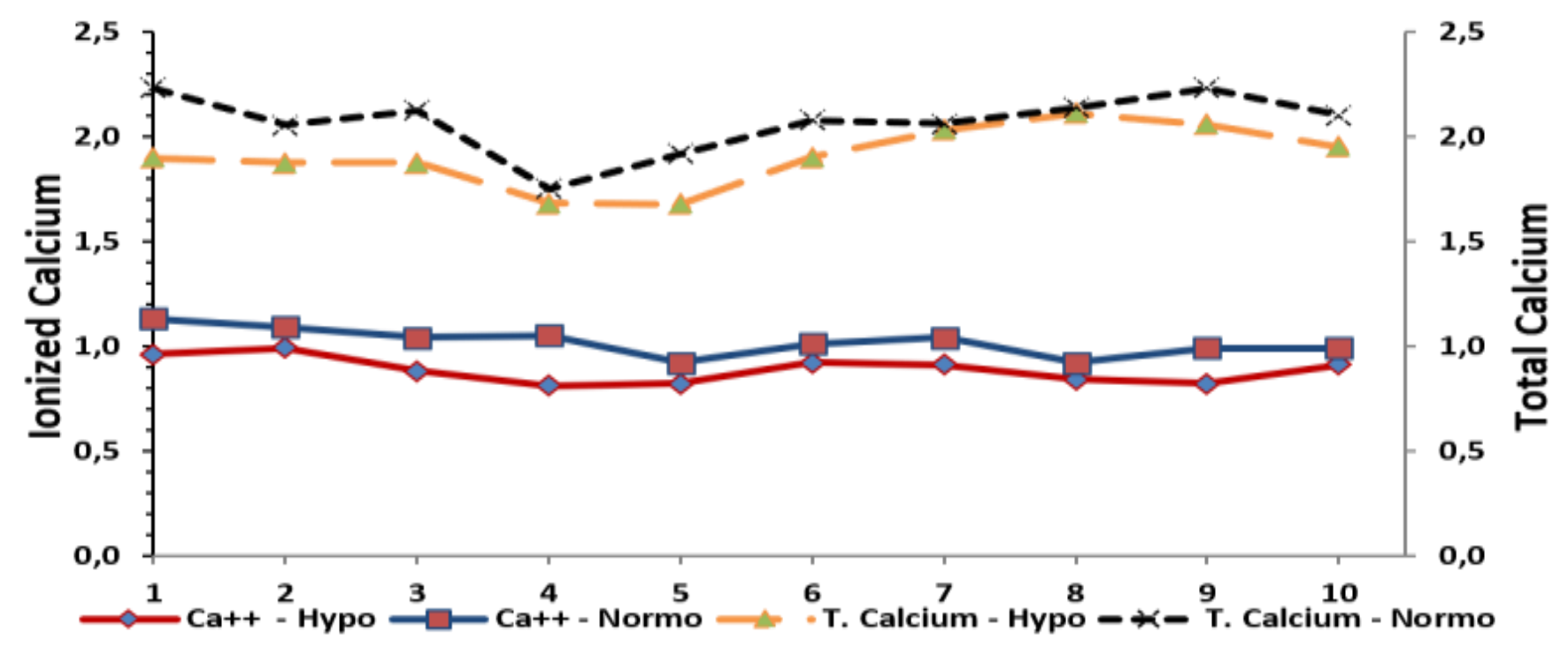

Source: Authors - Garanhuns Bovine Clinic, Federal Rural University of Pernambuco (2021).

Figure 1 clearly shows that the behavior of ionized calcium differs from total calcium throughout the experimental times in both groups, which justifies the low correlation between these variables. 


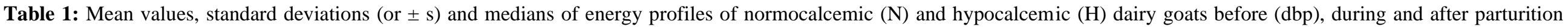
(dap).

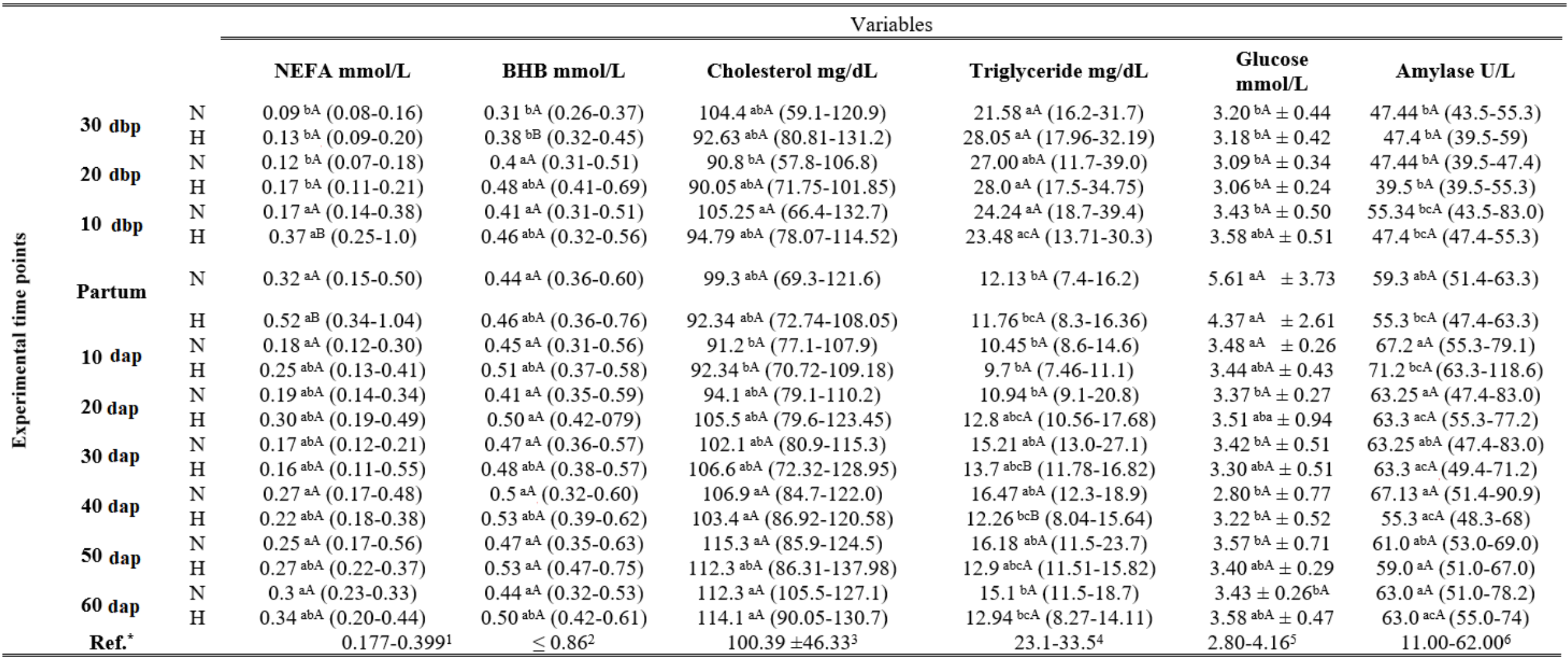

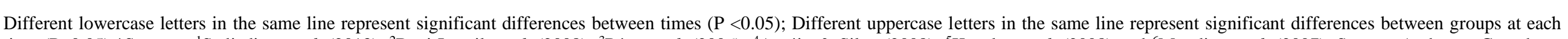

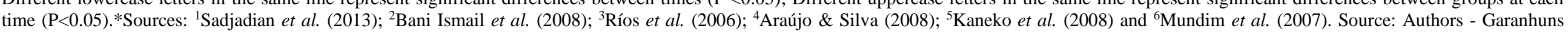
Bovine Clinic, Federal Rural University of Pernambuco (2021). 
The Table 1 shows that at the time of delivery, the serum concentration of $\mathrm{Ca}^{++}$decreased in both groups, being more pronounced in G1 than in G2, while blood glucose showed an increase in both groups compared to the other experimental moments. the other variables of the energy profile will be addressed in the Discussion.

The most relevant values with more direct metabolic relationships, such as BHB and NEFA, insulin and glucose, total protein and albumin, and the relationship of $\mathrm{Ca}^{++}$to triglycerides, amylase, $\beta$-hydroxybutyrate and NEFA are represented in Figs. 2, 3, 4 and 5. Was observed of the association between $\mathrm{Ca}^{++}$ions and other variables revealed the biological importance of $\mathrm{Ca}^{++}$to the variables as follows: strongly positive with triglycerides $(\mathrm{r}=0.75)$ in $\mathrm{G} 1$ and moderately positive $(\mathrm{r}=0.59)$ in G2; strongly negative with $\beta$-hydroxybutyrate $(r=-0.78)$ and amylase $(r=-0.90)$ in $G 2$; and moderately negative with NEFA $(\mathrm{r}=-0.56), \beta$-hydroxybutyrate $(\mathrm{r}=-0.49)$ and amylase $(\mathrm{r}=-0.53)$ in $\mathrm{G} 1$ and in G2 with NEFA $(\mathrm{r}=-0.53)$. Values and differences among the experimental time points and between the groups for energy, protein, enzyme and mineral variables and the hormone profiles are described in tables 2, 3, 4 and 5, respectively.

Table 2: Mean values, medians and confidence intervals of protein profiles of normocalcemic $(\mathrm{N})$ and hypocalcemic $(\mathrm{H})$ dairy goats before (dbp), during and after parturition (dap).

\begin{tabular}{|c|c|c|c|c|c|c|}
\hline & & & & Var & & \\
\hline & & & Total Protein g/dL & Albumin g/dL & Urea mg/dL & Creatinine $\mathbf{U} / \mathrm{L}$ \\
\hline & & $\mathrm{N}$ & $7.31^{\mathrm{bA}}(6.7-8.04)$ & $2.9^{\mathrm{aA}}(2.71-3.13)$ & $53.5^{\mathrm{bA}}(26.0-65.3)$ & 0.64 acA $(0.57-0.72)$ \\
\hline & 5000 & $\mathrm{H}$ & $7.37 \mathrm{bA}(7.1-7.87)$ & $2.72 \mathrm{abA}(2.37-2.85)$ & $35.9^{\mathrm{bA}}(28.7-52.6)$ & $0.76^{\mathrm{aB}}(0.67-0.88)$ \\
\hline & & $\mathrm{N}$ & $6.99 \mathrm{bA}(6.22-7.61)$ & $2.61^{\mathrm{bA}}(2.51-2.80)$ & $26.9^{\mathrm{bA}}(22.1-43.2)$ & $0.59 \mathrm{abcA}(0.53-0.71)$ \\
\hline & $20 \mathrm{Cop}$ & $\mathrm{H}$ & $7.01(6.29-7.85)^{\mathrm{bA}}$ & $2.5^{\mathrm{bA}}(2.21-2.77)$ & $47.6^{\mathrm{bA}}(25.8-57.1)$ & $0.69^{\mathrm{abB}}(0.58-0.84)$ \\
\hline & & $\mathrm{N}$ & $6.68^{\mathrm{bA}}(6.15-7.63)$ & $2.67 \mathrm{bA}(2.45-2.84)$ & $35.0^{\mathrm{bA}}(28.5-47.5)$ & $0.61^{\mathrm{abcA}}(0.57-0.68)$ \\
\hline & 10 & $\mathrm{H}$ & $7.12^{\mathrm{bA}}(6.43-7.95)$ & $2.54 \mathrm{bA}(2.25-2.83)$ & $58.0^{\mathrm{bA}}(46-65.2)$ & $0.63 \mathrm{abB}(0.58-0.78)$ \\
\hline & $\mathbf{D}$ & $\mathrm{N}$ & $7.00(6.28-8.24){ }^{\mathrm{bA}}$ & $2.66^{\mathrm{bA}}(2.38-2.99)$ & $47.9^{\text {bcA }}(40.6-57.2)$ & 0.63 aA $(0.63-0.74)$ \\
\hline 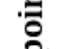 & & $\mathrm{H}$ & $7.04^{\mathrm{bA}}(6.49-7.68)$ & $2.62^{\mathrm{bA}}(2.32-2.72)$ & $54.0 \mathrm{abA}(35.3-84.5)$ & $0.64 \mathrm{abB}(0.53-0.79)$ \\
\hline$\stackrel{0}{E}$ & & $\mathrm{~N}$ & 7.85 acdA $(7.13-8.44)$ & $2.64 \mathrm{bA}(2.39-2.95)$ & 56.0 acA $(46.2-70.0)$ & $0.52^{\mathrm{bA}}(0.48-0.56)$ \\
\hline$=$ & 100 & $\mathrm{H}$ & $7.47^{\mathrm{bA}}(7.12-7.97)$ & $2.64^{\mathrm{bA}}(2-2.81)$ & 63.7 abA $(50.9-71)$ & $0.56^{\mathrm{bcB}}(0.52-0.64)$ \\
\hline 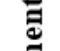 & $2+2>$ & $\mathrm{N}$ & 8.22 acA $(7.51-8.42)$ & $2.75 \mathrm{abA}(2.63-2.91)$ & $69.8^{\text {acA }}(41.9-77.9)$ & 0.52 bcA $(0.46-0.60)$ \\
\hline$F$ & 20 oap & $\mathrm{H}$ & 8.29 abcA $(7.82-8.48)$ & $2.75 \mathrm{abA}(2.38-2.90)$ & $66.0 \mathrm{abA}(52.4-80.6)$ & $0.57^{\mathrm{bB}}(0.52-0.67)$ \\
\hline 党 & & $\mathrm{N}$ & $8.36^{\mathrm{aA}}(7.78-8.7)$ & $2.90^{\mathrm{aA}}(2.66-3.15)$ & 63.8 acA $(40.0-76.3)$ & 0.53 bcA $(0.49-0.58)$ \\
\hline & & $\mathrm{H}$ & 8.53 acA $(8.36-8.75)$ & $2.88^{\mathrm{aA}}(2.74-3.06)$ & $63.7 \mathrm{aA}(51.4-96.5)$ & $0.61^{\mathrm{bB}}(0.53-0.67)$ \\
\hline & & $\mathrm{N}$ & 7.73 acA $(7.34-8.93)$ & $2.94 \mathrm{aA}(2.81-3.04)$ & 70.9 acA $(53.8-83.0)$ & $0.58^{\mathrm{bA}}(0.44-0.62)$ \\
\hline & & $\mathrm{H}$ & $8.28^{\mathrm{cA}}(8.12-8.44)$ & $2.75 \mathrm{abA}(2.54-3.05)$ & $76.5^{\mathrm{aA}}(60.9-98.3)$ & $0.61^{\mathrm{bA}}(0.52-0.73)$ \\
\hline & & $\mathrm{N}$ & $7.86^{\mathrm{acdA}}(7.50-8.23)$ & $2.67 \mathrm{abA}(2.57-3.03)$ & 75.5 aA $(45.2-94.1)$ & $0.55 \mathrm{abcA}(0.53-0.63)$ \\
\hline & & $\mathrm{H}$ & 7.81 abcA $(7.47-8.47)$ & $2.69^{\mathrm{abA}}(2.61-2.96)$ & $75.2^{\text {aA }}(58.4-96.5)$ & $0.61^{\mathrm{bA}}(0.54-0.68)$ \\
\hline & & $\mathrm{N}$ & 8.02 acdA $(7.52-8.30)$ & $2.78 \mathrm{abA}(2.67-2.96)$ & 77.7 aA $(64.3-92.2)$ & $0.61^{\mathrm{abcA}}(0.53-0.68)$ \\
\hline & & $\mathrm{H}$ & 8.09 acA $(7.56-8.70)$ & $2.74 \mathrm{abA}(2.59-2.92)$ & $77.7 \mathrm{aA}(57.2-90.2)$ & $0.61^{\mathrm{bA}}(0.58-0.70)$ \\
\hline & Ref. * & & $6.4-7.0^{1}$ & $3.07 \pm 0.41^{2}$ & $40.78-54.61^{3}$ & $0.8-8.9^{1}$ \\
\hline
\end{tabular}

Different lowercase letters in the same line represent significant differences between times $(\mathrm{P}<0.05)$; Different uppercase letters in the same line represent significant differences between groups at each time $(\mathrm{P}<0.05)$. *Sources: 1Kaneko et al. (2008); 2Mundim et al. (2007) and 3Sadjadian et al. (2013). Source: Authors - Garanhuns Bovine Clinic, Federal Rural University of Pernambuco (2021). 
In Table 2, the concentrations of total protein, albumin and urea were lower before delivery compared to delivery in both groups, while creatinine concentrations did not vary significantly between groups or between moments. However, the reasons for the changes in the concentrations of these variables and their relationship with ionizable calcium will be discussed later.

Figure 2: Median values of $\beta$-hydroxybutyrate (BHB) and nonesterified fatty acids (NEFA) in hypocalcemic and normocalcemic dairy goats during the transitional period.

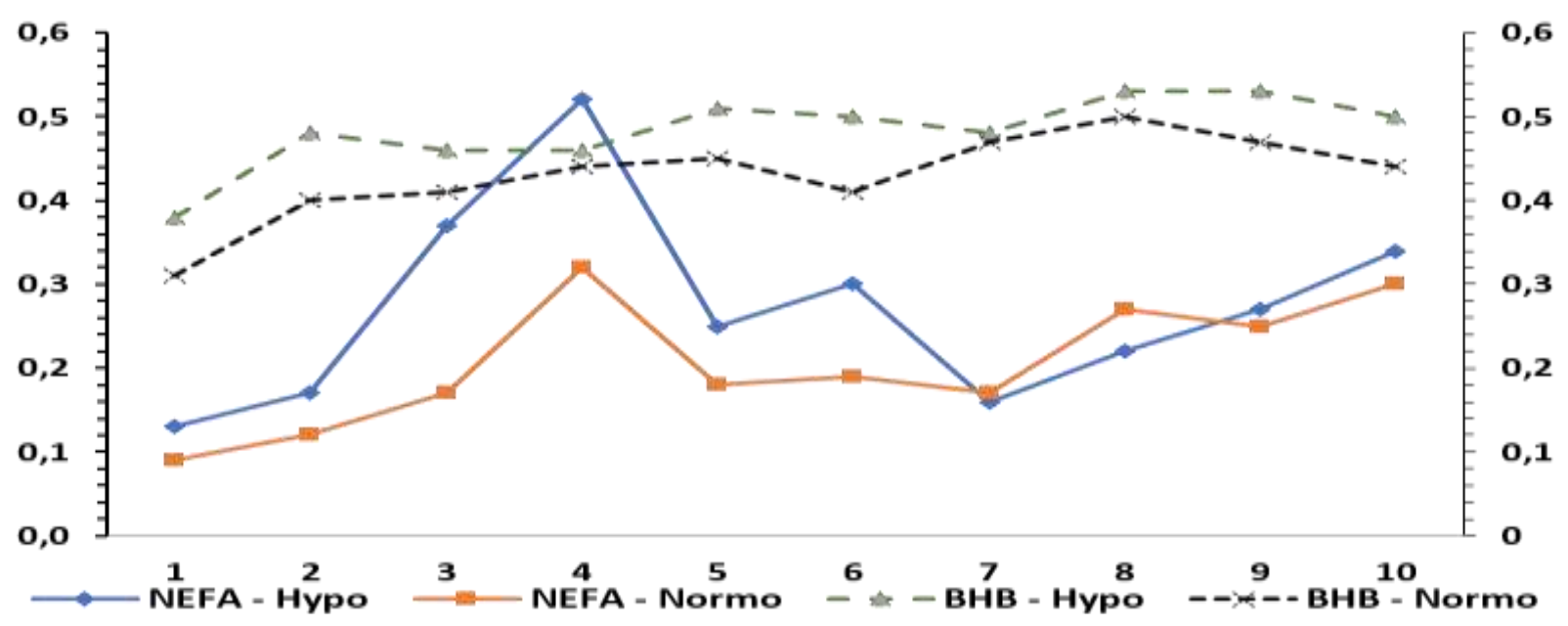

Source: Authors - Garanhuns Bovine Clinic, Federal Rural University of Pernambuco (2021).

In Figure 2 we observe that there is an increasing rate of adipose tissue mobilization, an increase in NEFA, during the prepartum period until the time of delivery and that this mobilization continues in the postpartum period at a lower intensity, however the NEFA values do not return at the lowest prepartum values during the first 60 days postpartum. In addition, throughout the prepartum period and up to 20 days postpartum, G1 presented NEFA values higher than G2. Although there is an increase in serum BHB concentrations at delivery and after delivery in both groups, it is noted that G1 had higher concentrations of BHB than $\mathrm{G} 2$ demonstrating the interference of $\mathrm{Ca}^{++}$in the energy profile. 
Table 3: Mean values, medians and confidence intervals of enzymatic profiles of normocalcemic $(\mathrm{N})$ and hypocalcemic $(\mathrm{H})$ dairy goats before (dbp), during and after parturition (dap).

\begin{tabular}{|c|c|c|c|c|c|}
\hline \multirow{23}{*}{ 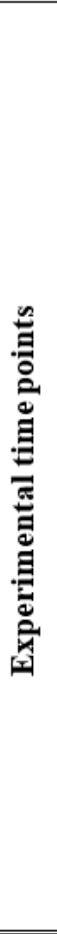 } & & & & Variables & \\
\hline & \multirow{3}{*}{30 dbp } & & AST (U/L) & GGT (U/L) & CK (U/L) \\
\hline & & $\mathrm{N}$ & $99.5^{\mathrm{abA}}(83.8-120.5)$ & $53.6 \mathrm{abA}(38.3-61.2)$ & $172.4^{\mathrm{bA}}(121.4-230.8)$ \\
\hline & & $\mathrm{H}$ & $99.5^{\mathrm{aA}}(85.1-104.8)$ & $53.6^{\mathrm{bA}}(40.2-61.2)$ & $99.9 \mathrm{abA}(54.6-193.5)$ \\
\hline & \multirow{2}{*}{20 dbp } & $\mathrm{N}$ & $83.8^{\mathrm{bA}}(78.6-99.5)$ & $45.9^{\mathrm{bA}}(30.6-53.6)$ & $149.8^{\mathrm{abA}}(112.3-218.6)$ \\
\hline & & $\mathrm{H}$ & 89.1 aA $(74.6-120.5)$ & $45.9^{\mathrm{bA}}(32.5-59.3)$ & $194.3^{\mathrm{abA}}(149.8-213.9)$ \\
\hline & \multirow{2}{*}{10 dbp } & $\mathrm{N}$ & $83.8^{\mathrm{bA}}(70.7-102.2)$ & $45.9 \mathrm{abA}(42.1-53.6)$ & $194.3^{\mathrm{abA}}(133.6-255.0)$ \\
\hline & & $\mathrm{H}$ & $78.6^{\mathrm{aA}}(69.4-104.8)$ & $45.9 \mathrm{abA}(40.2-66.9)$ & $145.7 \mathrm{abA}(121.4-273.2)$ \\
\hline & \multirow{2}{*}{ Partum } & $\mathrm{N}$ & $73.33^{\mathrm{bA}}(68.1-94.3)$ & $49.7 \mathrm{abA}(45.9-68.9)$ & $157.9^{\mathrm{abA}}(133.6-218.6)$ \\
\hline & & $\mathrm{H}$ & $83.8^{\text {aA }}(73.3-108.8)$ & $53.6^{\mathrm{abA}}(45.9-66.9)$ & $145.7^{\mathrm{bA}}(121.4-170)$ \\
\hline & \multirow{2}{*}{10 dap } & $\mathrm{N}$ & $99.5^{\mathrm{abA}}(78.6-128.4)$ & $53.6 \mathrm{abA}(45.9-68.9)$ & $170.0^{\mathrm{bA}}(121.4-194.3)$ \\
\hline & & $\mathrm{H}$ & $94.3^{\mathrm{aA}}(85.1-112.6)$ & $53.6^{\mathrm{abA}}(40.2-66.9)$ & $170.0^{\mathrm{bA}}(121.4-212.5)$ \\
\hline & \multirow{2}{*}{20 dap } & $\mathrm{N}$ & $104.8^{\mathrm{abA}}(78.6-165.0)$ & $53.6^{\mathrm{aA}}(45.9-91.8)$ & $218.6^{\mathrm{abA}}(145.7-242.9)$ \\
\hline & & $\mathrm{H}$ & $104.8 \mathrm{aA}(86.4-113.9)$ & $61.2^{\mathrm{aA}}(40.2-76.5)$ & $218.6^{\mathrm{abA}}(151.8-236.8)$ \\
\hline & \multirow{2}{*}{30 dap } & $\mathrm{N}$ & $89.0^{\mathrm{abA}}(73.3-133.6)$ & $53.6^{\mathrm{aA}}(45.9-76.5)$ & $206.5^{\mathrm{abA}}(145.7-242.9)$ \\
\hline & & $\mathrm{H}$ & $110^{\mathrm{aA}}(90.4-120.5)$ & $68.9^{\mathrm{aA}}(45.9-89.9)$ & $145.7 \mathrm{abA}(127.5-236.8)$ \\
\hline & \multirow{2}{*}{40 dap } & $\mathrm{N}$ & 107.4 aA $(89.0-149.3)$ & $53.4^{\mathrm{aA}}(45.9-88.0)$ & $224.7 \mathrm{abA}(157.9-279.3)$ \\
\hline & & $\mathrm{H}$ & $110^{\mathrm{aA}}(94.3-144.1)$ & $53.6^{\mathrm{aA}}(47.8-80.3)$ & $174.7 \mathrm{abA}(145.7-243.4)$ \\
\hline & \multirow{2}{*}{50 dap } & $\mathrm{N}$ & $107.4^{\mathrm{abA}}(68.2-180.8)$ & $61.2^{\mathrm{aA}}(45.9-84.2)$ & $224.7 \mathrm{abA}(112.3-299.6)$ \\
\hline & & $\mathrm{H}$ & $94.3^{\mathrm{aA}}(79.8-122)$ & $65.7^{\mathrm{aA}}(47.8-74.6)$ & $224.7 \mathrm{abA}(162.3-274.6)$ \\
\hline & \multirow{2}{*}{60 dap } & $\mathrm{N}$ & $104.7^{\mathrm{abA}}(81.2-120.5)$ & $61.2^{\mathrm{aA}}(45.9-80.3)$ & $243.9^{\mathrm{aA}}(162.3-287.1)$ \\
\hline & & $\mathrm{H}$ & $115.2^{\mathrm{aA}}(106.1-120.5)$ & $61.2^{\mathrm{aA}}(53.6-76.5)$ & $224.7^{\mathrm{aA}}(195.7-318.3)$ \\
\hline & Ref. $^{*}$ & & $167-513^{1}$ & $20-56^{1}$ & $100-250^{2}$ \\
\hline
\end{tabular}

Different lowercase letters in the same line represent significant differences between times $(\mathrm{P}<0.05)$; Different uppercase letters in the same line represent significant differences between groups at each time $(\mathrm{P}<0.05)$. ${ }^{*}$ Sources: ${ }^{1}$ Kaneko et al. $(2008)$ and ${ }^{2}$ Kannan et al. $(2000)$. Source: Authors - Garanhuns Bovine Clinic, Federal Rural University of Pernambuco (2021).

Table 3 shows that the AST and GGT enzymes are always within normal values in both groups and that CK presented an increase in its concentrations in the postpartum period in both groups, which may be related to the increase in muscular activity of the animals due to the intensification of their handling after parturition.

Figure 3: Median values of insulin and glucose in hypocalcemic and normocalcemic dairy goats during the transitional period.

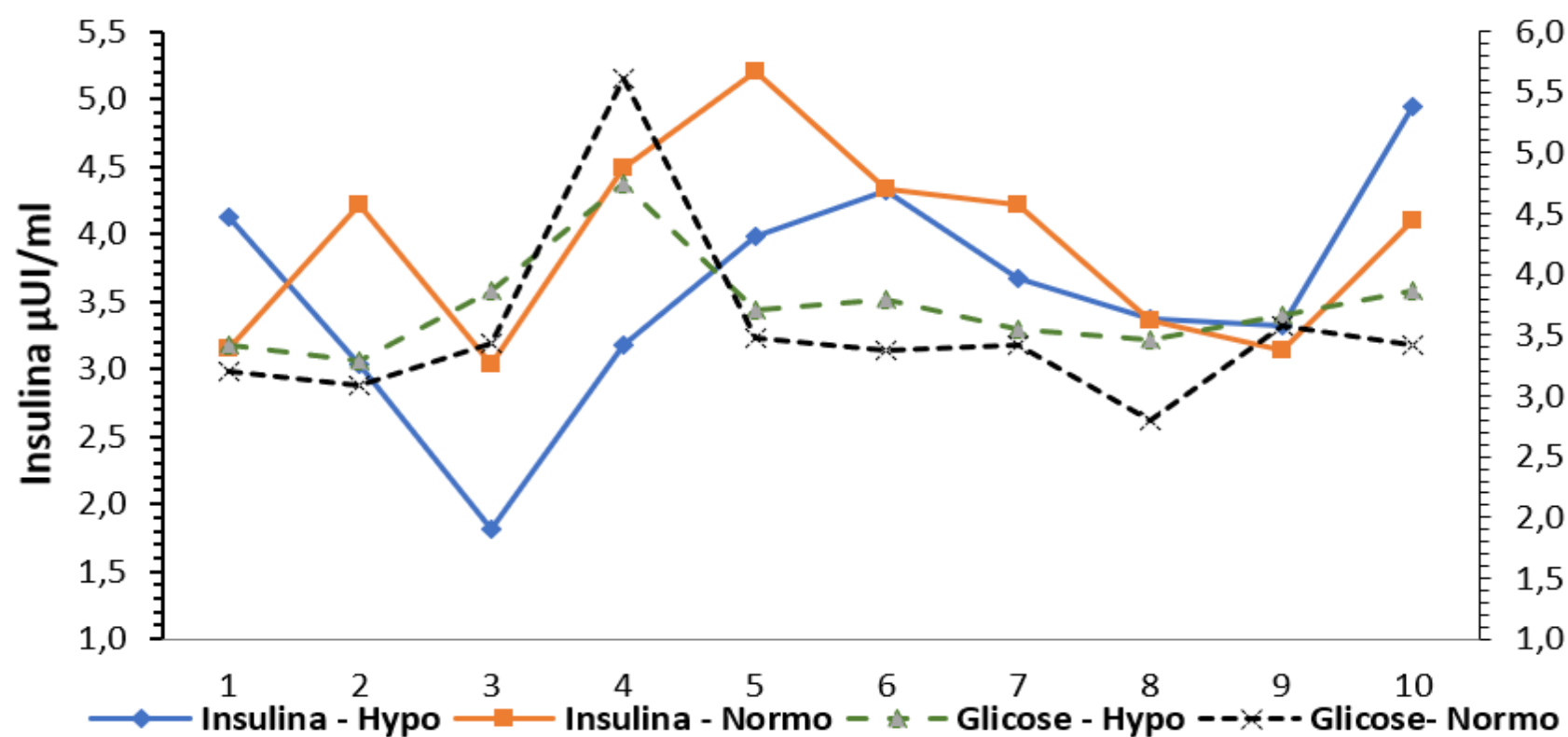

Source: Authors - Garanhuns Bovine Clinic, Federal Rural University of Pernambuco (2021). 
Figure 3 shows that G1 had lower serum insulin concentrations compared to G2 from 20dbp to 10dap, which demonstrates the strong influence of $\mathrm{Ca}^{++}$on insulin release from the pancreas. This directly influenced the serum glucose concentrations that in G1 tended to be higher than in G2 except at the time of delivery, which can be explained by the higher insulin resistance at delivery in G2 than in G1 due to a better homeorrhetic adjustment.

Figure 4: Median values of total protein and albumin in hypocalcemic and normocalcemic dairy goats during the transitional period.

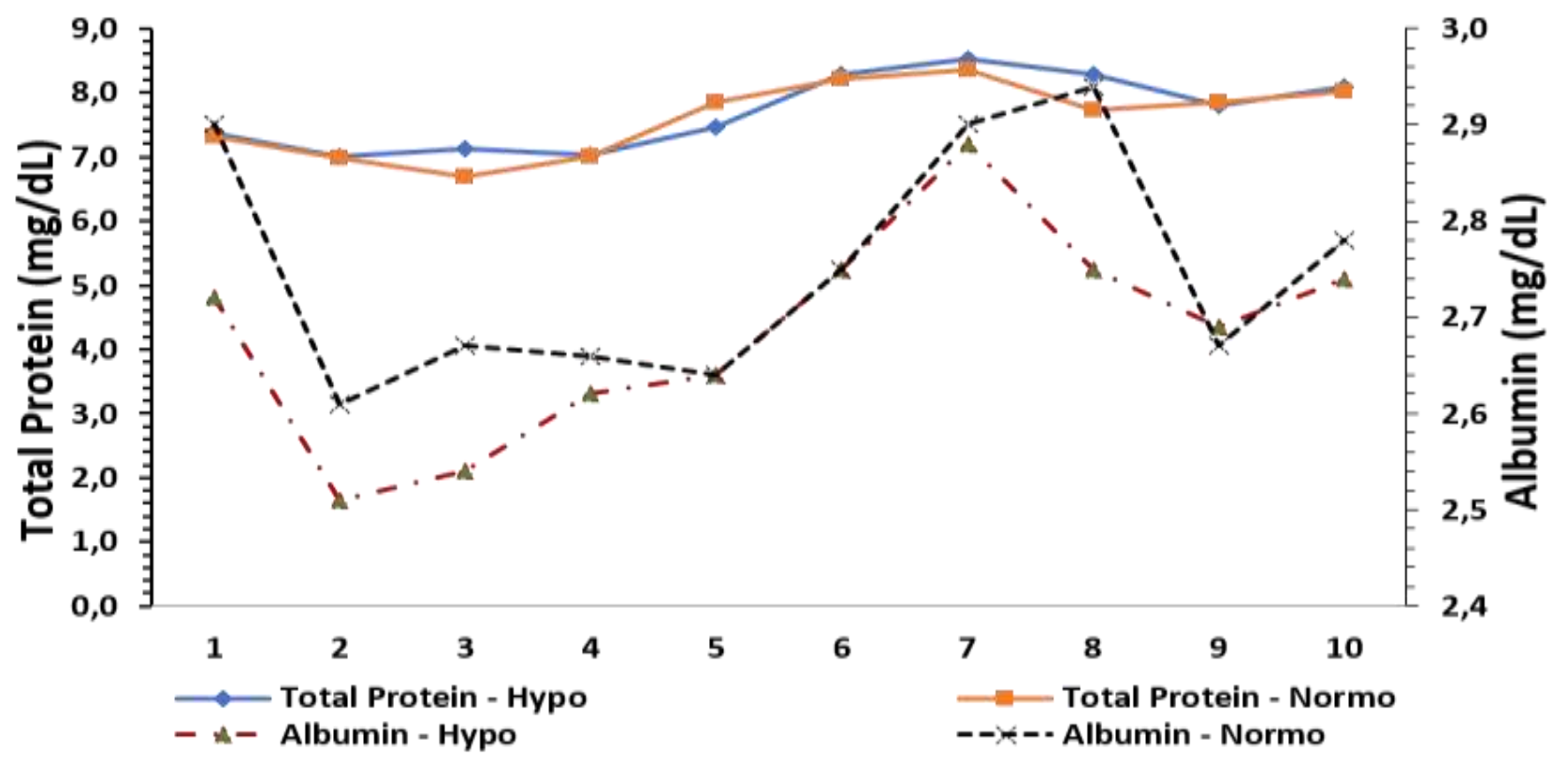

Source: Authors - Garanhuns Bovine Clinic, Federal Rural University of Pernambuco (2021).

Figure 4, shows that there is a reduction in serious concentrations of total protein and especially albumin in late pregnancy until delivery. After delivery there is an increase in total protein and especially in albumin, but both before delivery and after 30dap, albumin values in G1 are lower than in G2. 


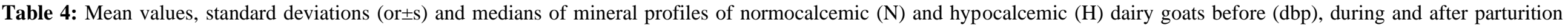
(dap).

\begin{tabular}{|c|c|c|c|c|c|c|c|c|c|}
\hline \multirow{23}{*}{ 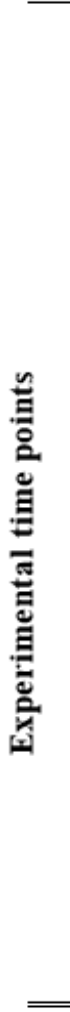 } & \multirow{4}{*}{30 dpb } & & \multirow[b]{2}{*}{$\mathrm{Ca}^{++} \mathrm{mmol} / \mathrm{L}$} & \multirow[b]{2}{*}{$\begin{array}{c}\text { Total Calcium } \\
\text { mmol/L }\end{array}$} & \multicolumn{4}{|c|}{ Variables } & \multirow[b]{2}{*}{$\begin{array}{c}\text { Potassium } \\
\text { mmol/L }\end{array}$} \\
\hline & & & & & Phosphor mmol/L & $\begin{array}{c}\text { Magnesium } \\
\mathrm{mmol} / \mathrm{L}\end{array}$ & Chlorides $\mathrm{mmol} / \mathrm{L}$ & Sodium mmol/L & \\
\hline & & $\mathrm{N}$ & $1.13 \mathrm{bcA} \pm 0.14$ & $2.23 \mathrm{bA} \pm 0.28$ & $1.96 \mathrm{abA}(1.56-2.25)$ & 0.99 bA $(0.90-1.22)$ & $117.4 \mathrm{aA}(114.5-119.6)$ & 151 acA $(148.0-152.0)$ & $4.4^{\mathrm{bA}}(4.2-4.75)$ \\
\hline & & $\mathrm{H}$ & $0.96 \mathrm{aB} \pm 0.16$ & $1.9 \mathrm{abcdA} \pm 0.4$ & $2.16^{\mathrm{aA}}(1.90-2.65)$ & $1.36 \mathrm{abA}(1.14-1.54)$ & $117.6 \mathrm{aA}(112.5-124.6)$ & $149 \mathrm{aA}(147-151)$ & $4.5 \mathrm{aA}(4.2-4.7)$ \\
\hline & & $\mathrm{N}$ & $1.09 \mathrm{abA} \pm 0.16$ & $2.06^{\mathrm{bA}} \pm 0.14$ & $2.15 \mathrm{abA}(1.93-2.53)$ & $1.34 \mathrm{aA}(1.29-1.44)$ & $119.4 \mathrm{aA}(113.7-123.9)$ & $150 \mathrm{abcA}(146.0-156.5)$ & $5.0 \mathrm{ab} A(4.55-5.3)$ \\
\hline & 20 аро & $\mathrm{H}$ & $0.98 \mathrm{abB} \pm 0.13$ & $1.9 \mathrm{abcdB} \pm 0.22$ & $2.67 \mathrm{aA}(2.15-2.99)$ & $1.47 \mathrm{aA}(1.33-1.62)$ & $116^{\mathrm{aA}}(113.9-121.4)$ & 149 aA $(146-151)$ & $4.9 \mathrm{bA}(4.7-5.1)$ \\
\hline & & $\mathrm{N}$ & $1.04 \mathrm{abA} \pm 0.14$ & $2.13^{\mathrm{bA}} \pm 0.14$ & $2.26 \mathrm{abA}(1.93-2.53)$ & $1.06^{\mathrm{aA}}(0.97-1.24)$ & $114.7 \mathrm{aA}(112.0-120.5)$ & 148 abca $(146.0-151.0)$ & $5.0 \mathrm{abA}(4.75-5.15)$ \\
\hline & 10 dpb & $\mathrm{H}$ & $0.88^{\mathrm{abb}} \pm 0.14$ & $1.87 \mathrm{abcdB} \pm 0.22$ & $2.59 \mathrm{aA}(1.93-2.99)$ & $1.25 \mathrm{abA}(0.96-1.71)$ & $117.7 \mathrm{aA}(113.2-121.5)$ & $148 \mathrm{aA}(145-150)$ & $4.8 \mathrm{abA}(4.5-5.0)$ \\
\hline & Partum & $\mathrm{N}$ & $1.05 \mathrm{abA} \pm 0.13$ & $1.75 \mathrm{aA} \pm 0.21$ & $2.03 \mathrm{abA}(1.60-2.54)$ & $0.82 \mathrm{bA}(0.72-1.05)$ & 119 acA $(115.2-124.6)$ & $151 \mathrm{aA}(148.5-154.0)$ & $4.6^{\mathrm{bA}}(4.35-4.9)$ \\
\hline & & $\mathrm{H}$ & $0.81^{\mathrm{bE}} \pm 0.15$ & $1.68 \mathrm{abA} \pm 0.16$ & $2.39 \mathrm{aA}(2.04-2.95)$ & $1.18^{\mathrm{bB}}(0.84-1.38)$ & 119 aA $(111.8-121)$ & $151 \mathrm{aA}(150-154)$ & $4.6 \mathrm{abA}(4.5-5.0)$ \\
\hline & & $\mathrm{N}$ & $0.92^{\mathrm{acA}} \pm 0.1$ & $1.92 \mathrm{abA} \pm 0.24$ & $2.12 \mathrm{abA}(1.91-2.65)$ & $0.82 \mathrm{bA}(0.72-1.05)$ & $114 \mathrm{acA}(110.3-121.1)$ & $146.5^{\mathrm{aA}}(144.0-148.0)$ & $5.05 \mathrm{abA}(4.8-5.25)$ \\
\hline & 1 & $\mathrm{H}$ & $0.82 \mathrm{abB} \pm 0.15$ & $1.68 \mathrm{aB} \pm 0.19$ & 2.70 ab $(2.40-2.90)$ & $1.18^{\mathrm{bB}}(0.84-1.38)$ & $116.9 \mathrm{aA}(111.7-124.2)$ & $148 \mathrm{aA}(145-152)$ & $4.9 \mathrm{abA}(4.7-5.1)$ \\
\hline & & $\mathrm{N}$ & $1.01 \mathrm{aA} \pm 0.11$ & $2.08^{\mathrm{bA}} \pm 0.22$ & $2.30 \mathrm{abA}(1.84-2.58)$ & $1.23 \mathrm{aA}(1.06-1.54)$ & $116.8^{\mathrm{acA}}(109.9-121.9)$ & $147.0 \mathrm{aA}(146.0-148.5)$ & $4.8 \mathrm{abA}(4.6-5.35)$ \\
\hline & 20 dap & $\mathrm{H}$ & $0.92^{\mathrm{abB}} \pm 0.15$ & $1.9 \mathrm{abcB} \pm 0.21$ & $2.30 \mathrm{abA}(1.84-2.58)$ & $1.47 \mathrm{abA}(1.17-1.62)$ & $115.9 \mathrm{aA}(108.6-117.8)$ & $146 \mathrm{aA}^{3}(145-150)$ & $5.0 \mathrm{abA}(4.7-5.1)$ \\
\hline & & $\mathrm{N}$ & $1.04^{\mathrm{bA}} \pm 0.13$ & $2.06^{\mathrm{bA}} \pm 0.18$ & $1.89 \mathrm{bA}(1.57-2.13)$ & $1.22 \mathrm{abA}(1.09-1.26)$ & 119.9 aA $(114.3-129.7)$ & $146.0^{\mathrm{aA}}(143.5-147.5)$ & $4.55^{\mathrm{bA}}(4.4-5.0)$ \\
\hline & 30 оар & $\mathrm{H}$ & $0.91 \mathrm{abB} \pm 0.15$ & $2.06^{\mathrm{bA}} \pm 0.18$ & $2.16^{\mathrm{aA}}(1.67-2.46)$ & $1.28 \mathrm{abA}(1.15-1.30)$ & $112.5 \mathrm{aB}(107.9-117.5)$ & $146^{\mathrm{aA}}(143-147)$ & $4.4 \mathrm{bA}(4.2-4.7)$ \\
\hline & & $\mathrm{N}$ & $0.92 \mathrm{acA} \pm 0.12$ & $2.14^{\mathrm{bA}} \pm 0.20$ & $2.38 \mathrm{aA}(2.14-2.88)$ & $1.33 \mathrm{aA}(1.09-1.44)$ & 114.5 ar $(108.7-118.8)$ & $145.5 \mathrm{bA}(143.0-151.0)$ & $4.75 \mathrm{abA}(4.1-5.3)$ \\
\hline & dap & $\mathrm{H}$ & $0.84 \mathrm{abA} \pm 0.11$ & $2.1 \mathrm{bcdA} \pm 0.26$ & $2.55 \mathrm{aA}(2.0-3.30)$ & $1.48^{\mathrm{aba}}(1.28-1.55)$ & $112.7 \mathrm{cA}(108.4-119)$ & $144^{\mathrm{bA}}(142-148)$ & $4.7 \mathrm{abA}(4.4-5.3)$ \\
\hline & & $\mathrm{N}$ & $0.99 \mathrm{aA} \pm 0.15$ & $2.23^{\mathrm{bA}} \pm 0.24$ & $2.1 \mathrm{abA}(1.44-2.8)$ & $1.45^{\mathrm{aA}}(1.21-1.59)$ & 114 acA $(111.0-117.4)$ & $145.5^{\mathrm{bA}}(145.0-147.0)$ & $5.15 \mathrm{aA}(4.9-5.4)$ \\
\hline & s0 дар & $\mathrm{H}$ & $0.82^{\mathrm{abB}} \pm 0.12$ & $2.06^{b c d B} \pm 0.16$ & $2.55 \mathrm{aB}(2.35-3.38)$ & $1.48^{\mathrm{aA}}(1.41-1.64)$ & $110^{\mathrm{aA}}(108.6-115.2)$ & 145 bA (144-147) & $4.9 \mathrm{aA}(4.6-5.3)$ \\
\hline & & $\mathrm{N}$ & $0.99 \mathrm{aA} \pm 0.7$ & $2.1^{\mathrm{bA}} \pm 0.13$ & $1.97 \mathrm{abA}(1.73-2.3)$ & $1.39 \mathrm{aA}(1.27-1.51)$ & 114 acA $(110.3-116.2)$ & $149 \mathrm{aA}(145.5-151.0)$ & $4.85 \mathrm{abA}(4.7-5.2)$ \\
\hline & 60 dap & $\mathrm{H}$ & $0.92^{\mathrm{abA}} \pm 0.13$ & $1.9 \mathrm{abcdB} \pm 0.23$ & $2.46 \mathrm{aB}(2.30-2.92)$ & $1.35 \mathrm{abA}(1.23-1.55)$ & $117.2^{\mathrm{aA}}(113.5-117.4)$ & $148 \mathrm{aA}(146-149)$ & $4.6^{\mathrm{abB}}(4.4-4.8)$ \\
\hline & Ref.* & & $0.83 \pm 0.1^{2}$ & $2.23-2.93^{1}$ & $1.36-2.93^{1}$ & $0.31-1.48^{1}$ & $99-110.3^{1}$ & $142-155^{1}$ & $3.5-6.7^{1}$ \\
\hline
\end{tabular}

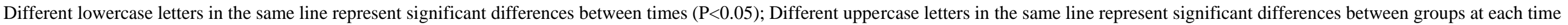
(P<0.05). *Sources: ${ }^{1}$ Kaneko et al. (2008) and ${ }^{2}$ Simplício et al. (2009). Source: Authors - Garanhuns Bovine Clinic, Federal Rural University of Pernambuco (2021). 
Table 5: Mean values, standard deviations (or \pm s) and medians of hormonal profiles for normocalcemic (N) and hypocalcemic (H) dairy go ats before (dbp), during and after parturition (dap).

\begin{tabular}{|c|c|c|c|c|}
\hline \multirow{23}{*}{ 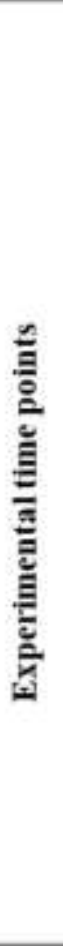 } & & & \multicolumn{2}{|c|}{ Variables } \\
\hline & \multirow{3}{*}{$30 \mathrm{dbp}$} & & Insulin $\mu \mathrm{UI} / \mathrm{ml}$ & Cortisol nmol/L \\
\hline & & $\mathrm{N}$ & $3.16^{\mathrm{aA}}(2.26-6.19)$ & $28.9^{\text {aA }}(19.3-59.9)$ \\
\hline & & $\mathrm{H}$ & $4.12^{\text {abA }}(2.4-4.97)$ & $37.5^{\text {ab. }}(19.6-47.1)$ \\
\hline & & $\mathrm{N}$ & $4.21^{\text {aA }}(2.70-4.74)$ & $38.1^{\text {tha }}(23.7-58.1)$ \\
\hline & $20 \mathrm{dbp}$ & $\mathrm{H}$ & $3.03^{\mathrm{abA}}(1.95-3.71)$ & $45.4^{\text {sbA }}(37.9-60.4)$ \\
\hline & & $\mathrm{N}$ & $3.03^{\text {aA }}(1.62-5.89)$ & $43.8^{\text {abA }}(25.6-52.5)$ \\
\hline & $10 \mathrm{dbp}$ & $\mathrm{H}$ & $1.82^{\mathrm{bA}}(0.89-3.16)$ & $42.6^{\text {abca }}(24.6-48.9)$ \\
\hline & & $\mathrm{N}$ & $4.49^{\mathrm{aA}}(2.31-7.94)$ & $60.1^{\mathrm{AA}}(18.7-72.3)$ \\
\hline & Partum & $\mathrm{H}$ & $3.18^{\mathrm{abA}}(2.50-3.64)$ & $59.3^{a c A}(35.9-81.0)$ \\
\hline & & $\mathrm{N}$ & $5.20^{\text {aA }}(3.52-6.84)$ & $40.1^{\text {abA }}(19.9-54.4)$ \\
\hline & 10 dap & $\mathrm{H}$ & $3.98^{\mathrm{AA}}(2.95-5,60)$ & $34.5^{\text {abca }}(24.6-42.4)$ \\
\hline & & $\mathrm{N}$ & $4.33^{2 A}(3.76-5.42)$ & $34.2^{\text {abA }}(17.2-48.5)$ \\
\hline & 20 dap & $\mathrm{H}$ & $4.32^{\mathrm{abA}}(3.28-5.0)$ & $25.6^{\text {bcA }}(12.2-35.1)$ \\
\hline & & $\mathrm{N}$ & $4.22^{\mathrm{aA}}(3.45-5.34)$ & $11.5^{\mathrm{bA}}(8.15-19.7)$ \\
\hline & 30 dap & $\mathrm{H}$ & 3.67 a $(3.2-5.92)$ & $16.6^{\mathrm{bA}}(11.0-19.2)$ \\
\hline & & $\mathrm{N}$ & $3.37^{a A}(2.59-4.7)$ & $42.0^{\mathrm{b} A \mathrm{~A}}(21.4-86.0)$ \\
\hline & 40 dap & $\mathrm{H}$ & $3.37^{\mathrm{abA}}(2.57-5.55)$ & $26.3^{\mathrm{abcA}}(15.2-46,8)$ \\
\hline & & $\mathrm{N}$ & $3.14^{2 A}(2.86-5.24)$ & $25.4^{\text {abA }}(13.0-47.7)$ \\
\hline & 50 dap & $\mathrm{H}$ & $3.32^{\mathrm{abA}}(2.27-4.38)$ & $20.7^{\mathrm{BA}}(9.8-38.1)$ \\
\hline & & $\mathrm{N}$ & $4.10^{2 \mathrm{~A}}(2.65-5.49)$ & $24.0^{2 b A}(16.7-39.4)$ \\
\hline & 60 dap & $\mathrm{H}$ & $4.94^{2 A}(2.95-6.50)$ & $29.5^{\text {bcA }}(16.0-40.0)$ \\
\hline & Ref. & & $8.00-24.53^{1}$ & $22.08-41.40^{2}$ \\
\hline
\end{tabular}

Different lowercase letters in the same line represent significant differences between times $(\mathrm{P}<0.05)$; Different uppercase letters in the same line represent significant differences between groups at each time $(\mathrm{P}<0.05)$. *Sources: ${ }^{1}$ Magistrelli and Rosi (2014) and ${ }^{2} \mathrm{He}$ et al. $(2015)$. Source: Authors - Garanhuns Bovine Clinic, Federal Rural University of Pernambuco (2021).

The insulin values listed in Table 5 have already been commented on in Figure 3. However, in relation to cortisol concentrations, it can be noted that in late pregnancy and especially in delivery there was an increase in this hormone in both groups. G1 had higher cortisol concentrations than G2 before delivery and in both groups there was a reduction in cortisol in the postpartum period. 
Figure 5: Graphical representation of the relationship of $\mathrm{Ca}^{++}$to triglycerides, amylase, $\beta$-hydroxybutyrate and NEFA in normocalcemic and hypocalcemic dairy goats during the transitional period.

Hypocalcemic - Triglycerides

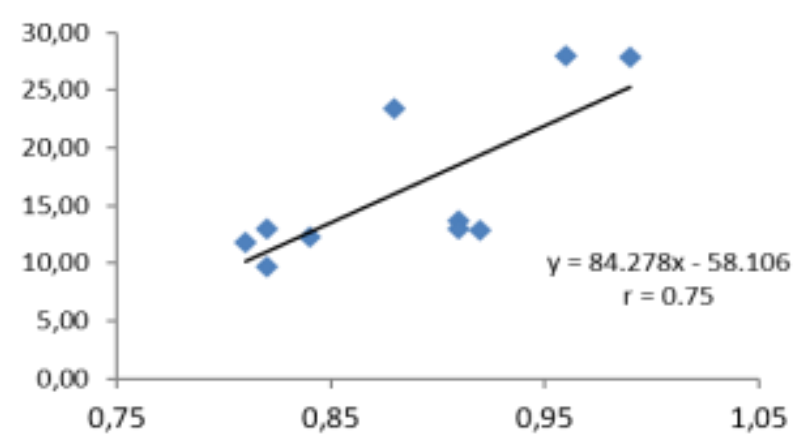

Normocalcemic - Triglycerides

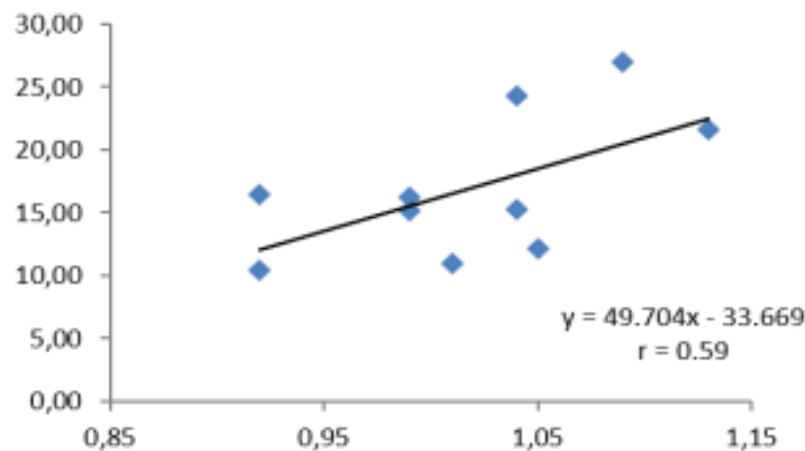

Hypocalcemic - Amylase

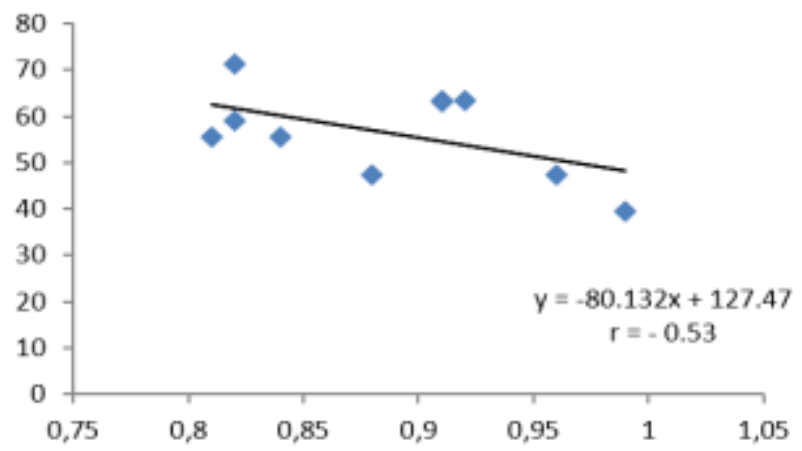

Normocalcemic - Amylase

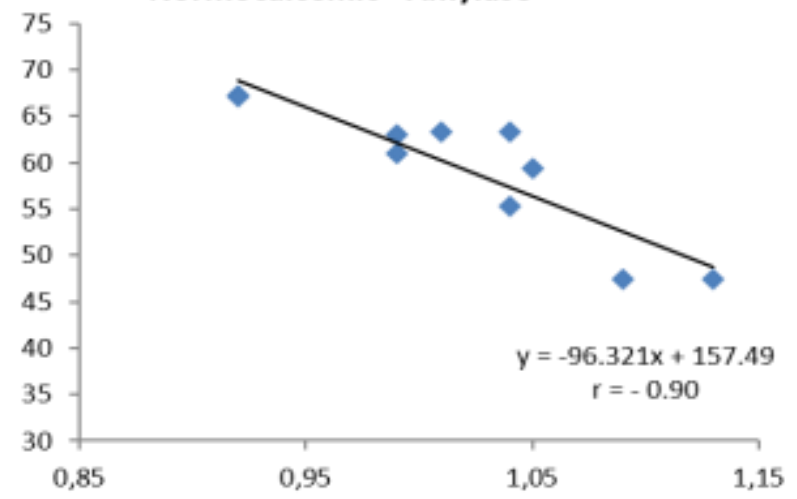

Hypocalcemic - BHB

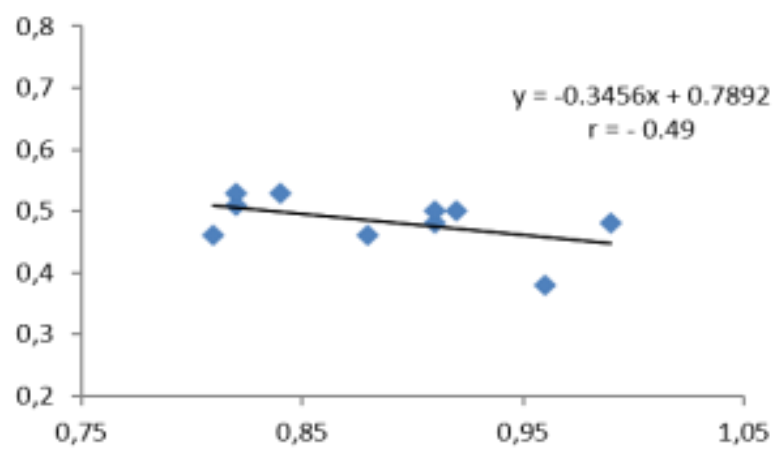

Normocalcemic - BHB

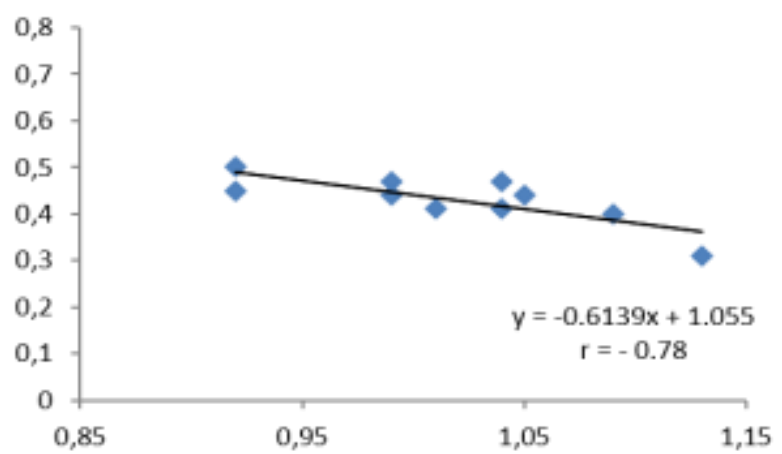

Normocalcemic - NEFA

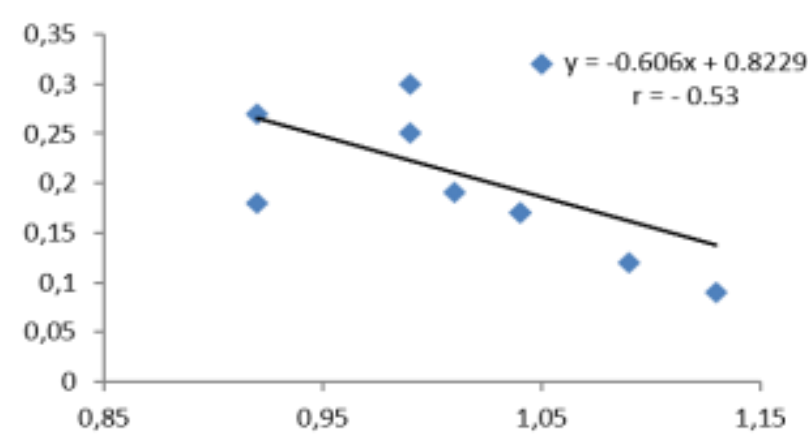

Hypocalcemic - NEFA

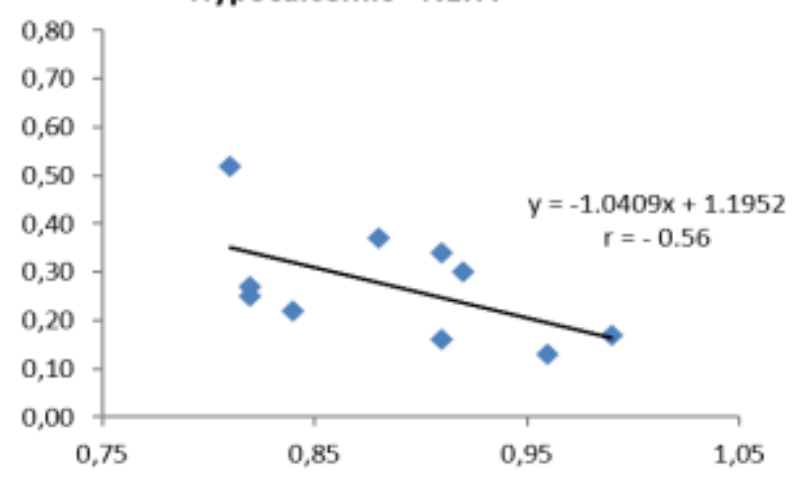

Source: Authors - Garanhuns Bovine Clinic, Federal Rural University of Pernambuco (2021). 
The correlation of $\mathrm{Ca}^{++}$with the energy profile variables whose graphs are shown in Figure 5 will be discussed in detail below, as well as the correlation between $\mathrm{Ca}++$ and protein and mineral profile variables.

\section{Discussion}

The changing ionized calcium levels observed over time can be explained by two factors: a lower dry matter intake near parturition (Grümmer, 1995; Bell, 1995; Goff \& Horst, 1997) and the increased demand for $\mathrm{Ca}^{++}$at parturition and at the beginning and peak of lactation in dairy goats (Goff \& Horst, 1997; Liesegang \& Risteli, 2005; Smith \& Sherman, 2009). As ionized calcium is the biologically active fraction of this mineral in animals (Kimura et al., 2006; Vieira, 2007, Santos, 2011), serum concentrations of this element are reduced with increased demand at lactation due to the movement of $\mathrm{Ca}^{++}$from the blood to the mammary glands (DeGaris \& Lean, 2008).

According to Goff and Horst (1997), Horst et al. (2005) and Santos (2011), the increase in $\mathrm{Ca}^{++}$demand in dairy cows can be better understood when considering that each liter of colostrum contains $2.3 \mathrm{~g}$ of $\mathrm{Ca}^{++}$; therefore, a cow producing $10 \mathrm{~L}$ of colostrum more than doubles its requirement of $\mathrm{Ca}^{++}$(43g/days) relative to a cow during the dry period (20g/days). This amount of $\mathrm{Ca}^{++}$in colostrum corresponds to nine times the total serum amount in a 600-kg cow. Similarly, Zambom et al. (2006) and Mundim et al. (2007) found an increased demand for $\mathrm{Ca}^{++}$and other nutrients in dairy goats at the beginning as well as the peak of lactation, occurring on average between 40 and 60 days after partum, a finding that was also observed in our research.

In addition, serum calcium levels are influenced, among other factors, by the amount of calcium in the diet (Schröder et al., 1997; Goff, 2006). In early lactation, dry matter intake is also decreased; consequently, changes in female physiology during the transitional period result in reduced $\mathrm{Ca}^{++}$intake. These changes contribute to a reduction in serum $\mathrm{Ca}^{++}$levels, activating the biological mechanisms responsible for the increase in serum $\mathrm{Ca}^{++}$(Smith \& Sherman, 2009; Santos, 2011). Data presented in the literature support the explanation of ionized calcium levels described in this paper.

With respect to larger AGNES values in G1 compared to G2, such results corroborate those reported by Sadjadian et al. (2013), who monitored the serum levels of this element in Saanen dairy goats 30 days before to 40 days after parturition; they noticed a gradual increase in the antepartum NEFA concentrations, with a peak $(0.40 \mathrm{mmol} / \mathrm{L})$ at parturition, as seen in G1 and $\mathrm{G} 2$, when the values were $0.52 \mathrm{mmol} / \mathrm{l}$ and $0.32 \mathrm{mmol} / \mathrm{L}$, respectively.

According to Bell (1995), this rise in NEFA occurs due to the association of three main factors: an increase in the energy demand for the initial production of colostrum and milk, parturition stress and reduced dry matter intake. This has been well characterized for this period in both cows and dairy goats (Grummer, 1995; Mundim et al., 2007).

The importance of these results is emphasized by a moderate correlation between NEFA and $\mathrm{Ca}^{++}$, which was corroborated by Reinhardt et al. (2011), who claimed a direct relationship between the NEFA and $\mathrm{Ca}^{++}$concentrations in dairy cows. When the $\mathrm{Ca}^{++} \geq 1 \mathrm{mmol} / \mathrm{L}$, the NEFA serum concentrations are lower, which means the normocalcemic cows have better energy balance than those with subclinical hypocalcemia. Thus, the results in this study suggest that normocalcemic dairy goats have better energy balance than those with subclinical hypocalcemia.

The BHB concentrations found in this work, as well as changes in levels of this variable over time, corroborate the findings of Ríos et al. (2006) and Sadjadian et al. (2013) in dairy goats and Van Der Drift et al. (2012) in dairy cattle. Those authors showed a significant increase in BHB values in early lactation (peak production) relative to the antepartum period. In this study, higher BHB concentrations were also observed in this period (40 dap).

The physiological explanation for these BHB concentrations was given by Santos (2011). This author affirmed that NEFA in the bloodstream increases due to the mobilization of lipids. NEFA is taken up by the liver and, as the liver is poor at 
exporting fatty acids in the form of very low-density lipoproteins (VLDL); fatty acids are esterified and deposited in the form of triglycerides until they are fully oxidized (upon entering the Krebs cycle), exported in the form of VLDL or transformed into ketone bodies, including BHB (Caldeira, 2005; Gonzáles \& Silva, 2006).

The reduction in food intake and dietary nutrients absorption can be explained by the higher values of BHB observed in G1. Because $\mathrm{Ca}^{++}$is important in nerve impulse transmission and in smooth and skeletal muscle contraction, where it acts as a second messenger, it controls adenosine triphosphate (ATP) release in the actin-myosin system (Horst et al., 1994; Goff, 2000; Schröder \& Breves, 2006; González \& Silva, 2017). Therefore, the reduced concentrations of this element in the serum are related to decreased gastrointestinal motility, appetite and the animal's displacement while searching for food (Oetzel, 1988; Smith \& Sherman, 2009). This explains why animals with some degree of hypocalcemia have a higher risk of developing hyperketonemia (Schlumbohm \& Harmeyer, 1990; Goff, 2006; Reinhardt et al., 2011).

A lesser correlation (moderate) exists between BHB and $\mathrm{Ca}^{++}$in the $\mathrm{G} 1$ than in the $\mathrm{G} 2$. This is because the serum concentration of this mineral in hypoglycemic goats decreases earlier during the antepartum period, whereas the BHB in this group also declines slightly during this period. The decline in BHB can be explained by the greater use of this element as an energy source, since there is a reduction in propionate due to decreased food intake (Grummer, 1995; Goff \& Horst, 1997; Goff, 2006).

The significantly higher triglyceride values in the prepartum period relative to parturition and postpartum are due to intense body fat mobilization. Because the liver is unable to partially (forming ketone bodies) or fully oxidize all NEFA, this product mobilizes, resulting in the transformation of excess NEFA into triglycerides. The lower concentration of this element in the postpartum period may be related to an increase in insulin and better hormone response by the target tissues, which increases the triglyceride uptake by circulating cells (Caldeira \& Portugal, 1991; Grummer, 1995; Gonzáles \& Silva, 2006).

A strong positive correlation was observed between the triglycerides and $\mathrm{Ca}^{++}$in $\mathrm{G} 1$, and a moderately positive correlation was observed in G2. This correlation reflects the high demand for these nutrients, which are more significant in parturition and postpartum and are proportional to milk production. Furthermore, it reflects the influence of $\mathrm{Ca}^{++}$on dry matter intake, which leads to the release of pancreatic hormones and therefore controls energy metabolism in dairy goats (Horst et al., 1994; Gupta et al., 2005; Goff, 2006; Schröder \& Breves, 2006; Smith \& Sherman, 2009).

The increase in postpartum cholesterol and the reduction in triglycerides found in this study were reported by Bennis $e t$ al. (1992) and Iriadam (2007) for dairy goats and by Basoglu et al. (1998) for dairy cows. Furthermore, Barbosa et al. (2009) suggested that the low prepartum cholesterol concentrations may result from the use of cholesterol by the fetus; progesterone and adrenal hormone synthesis may also reflect this use to a lesser extent. The cholesterol increase in the postpartum period may be associated with increased dry matter intake and energy concentration in the diet (Ríos et al., 2006).

The moderately negative correlation between $\mathrm{Ca}^{++}$and cholesterol in $\mathrm{G} 2$ can be explained by serum ionized calcium values that decline close to parturition in normocalcemic animals and immediately rise again without interfering with food intake, such as that which occurs in hypocalcemic animals (Goff \& Horst, 1997; Goff, 2006; Goff, 2008), thereby allowing cholesterol to increase in the postpartum period.

Amylase concentrations remained above the values described by Mundim et al. (2007) and Araújo \& Silva (2008) for goats. These concentrations are linked to a higher carbohydrate intake (Souto et al., 2013). Amylase is a metalloenzyme that is dependent on $\mathrm{Ca}^{++}$, and it acts in the intestine, hydrolyzing glucose polymers (starch, amylopectin and glycogen) in a 1,4 glycosidic link, producing maltose and dextrin (Kaneko et al., 2008). Because it is produced and released by the exocrine pancreas, its elevation appears to reflect good performance of pancreatic exocrine function. A decrease in amylase is not rare and is usually associated with animals that are subjected to diets low in starch (González \& Scheffer, 2002; Araujo \& Silva, 2008). 
Regarding the associations observed in this study, amylase showed a strongly negative relationship with the $\mathrm{Ca}^{++}$in $\mathrm{G} 1$ and only a moderately negative relationship with $\mathrm{Ca}^{++}$in G2. According to Araújo and Silva (2008), Kaneko et al. (2008) and Souto et al. (2013), this fact can be explained by the earlier and more marked reductions of $\mathrm{Ca}^{++}$in $\mathrm{G} 1$, which were associated with increased energy concentrates in the diet during the prepartum period and continued into the postpartum period, caused an increase in serum amylase.

The insulin behavior over the periods in this study was similar to the results obtained by Basoglu et al. (1998) in cows and by Lima et al. (2016) in sheep. Grummer (1995), McGuire et al. (1995), Basoglu et al. (1998) and De Koster \& Opsomer (2013) provided an explanation, indicating that the reduction in food intake in late pregnancy and parturition, which decreases the serum concentrations of gluconeogenesis precursors (glucose and propionate), are linked to high glucose demand by the fetus, which reduces insulin release from the pancreas and leads to a reduction in the serum levels of this hormone.

The largest reduction in insulin occurred in hypocalcemic goats, likely because of four main factors: the lower dry matter intake, the negative action of calcium on insulin release from pancreatic $\beta$ cells, the inhibitory effect of low calcium concentrations on hepatic gluconeogenesis and the increased insulin resistance by target tissues (Grummer, 1995; Goff \& Horst, 1997; Schlumbohm et al., 1997; Walz et al., 2007). The highest concentrations of NEFA in G1 and the negative influence of these concentrations on insulin secretion may be another compromising mechanism in the metabolism of this hormone (Reinhardt et al., 2011).

The highest serum cortisol concentrations detected in this study at parturition agree with the findings of El-Belely et al. (2000). Those authors, who studied sheep, found that the serum cortisol concentration was maintained at a constant level throughout gestation $(4.8 \pm 0.58 \mathrm{ng} / \mathrm{mL})$; however, a five-fold increase $(23.7 \pm 2.12 \mathrm{ng} / \mathrm{mL})$ occurred in the two days prior to calving. In cows, a significant increase also occurs in cortisol values at parturition (Horst \& Jorgensen, 1982). This element has been considered a good indicator of stress, and its powerful gluconeogenic effect appears to be its principal function in the peripartum period (Campos et al., 2009).

However, despite the increasing glycemia and the facilitation of lipolysis by increasing serum concentrations of NEFA (Huzzey et al., 2011), cortisol showed no negative correlation with $\mathrm{Ca}^{++}$, which does not agree with the results of Horst and Jorgensen (1982). Those authors found a cortisol elevation in cows and goats with clinical and subclinical hypocalcemia. This difference can be explained by the shorter analysis period ( 3 days before to 2.5 days postpartum) in those studies.

The glucose serum concentrations obtained in this study agree with the results reported by Santos et al. (2012) for sheep, by Grummer (1995) for cows and by Barbosa et al. (2009) for dairy goats. Those authors found higher values of this variable at parturition relative to the postpartum period, with different body condition scores (BCS) observed at calving. However, this finding is not consistent because Duehlmeier et al. (2013) found different glycemia behaviors in different sheep breeds; in one of the breeds, the glucose values did not differ throughout the transitional period or lactation.

The higher glucose concentrations observed at birth were explained by Russell \& Roussel (2007) and Kaneko et al. (2008), who surveyed glycemia behavior in ruminants and found that stressful situations induce hyperglycemia mediated by epinephrine and endogenous glucocorticoid release. Glucocorticoids increase gluconeogenesis and therefore blood glucose. Moreover, according to De Koster and Opsomer (2013), in late pregnancy and early lactation, insulin resistance occurs as a homeorhetic adaptation of these animals in order to prioritize glucose absorption by the fetus and mammary glands. Insulin resistance can contribute to a transient increase in the blood glucose levels at parturition. In dairy goats, a similar mechanism may be present.

The moderately negative correlation between glucose and $\mathrm{Ca}^{++}$found in this study disagrees with the findings of Schlumbohm and Harmayer (2003). In an experiment with sheep, they found that hypocalcemia induction results in a decline in plasma glucose concentrations at all stages of production. However, evidence exists that plasma calcium concentrations 
reduce the glucose utilization rate by tissues, which could help increase blood glucose. Interestingly, in hypocalcemic animals, insulin resistance is heightened, which may contribute to an increase in blood glucose levels (Schlumbohm \& Harmayer, 1990; Schlumbohm et al., 1997). Furthermore, hypocalcemia negatively affects insulin release by pancreatic $\beta$ cells because exocytosis of this hormone is impossible without $\mathrm{Ca}^{++}$(Walz et al., 2007).

Lower total serum protein concentrations were found in the prepartum period, which agrees with the findings of Balıkc1 et al. (2007) and Iriadam (2007), who observed declines in this element at the end of pregnancy in sheep and goats upon measuring the beginning of pregnancy, the middle of pregnancy and the postpartum period. Furthermore, the average values of total protein observed in the prepartum period of this study were within the values reported by Mundim et al. (2007). However, the concentrations of variables in the postpartum period were above the reference values in both groups (Kaneko et al., 2008).

Reductions in total protein at the end of pregnancy are due to an exponential increase in the demand for fetus formation and especially for colostrum immunoglobulin production (Santos et al., 2012). This requirement can be demonstrated quantitatively because the protein requirement of the fetus in cows during this period is approximately $998 \mathrm{~g} / \mathrm{d}$, and the demand for 10 liters of colostrum production is $1400 \mathrm{~g}$ (Bell, 1995).

This increased demand, which is associated with low food intake, is responsible for the reduction in total protein concentrations. In addition to the high demand for energy during this period, tissue protein mobilization and the formation of hepatic gluconeogenesis substrate by circulating and dietary proteins are occurring (Bell, 1995; Grummer, 1995; Bell \& Bauman, 1997; Goff \& Horst, 1997).

The moderately negative correlation between total protein and $\mathrm{Ca}^{++}$in $\mathrm{G} 2$ occurred because of the significant decline in $\mathrm{Ca}^{++}$, especially in the early postpartum period, when protein concentrations begin to rise. The lower demand for proteins after delivery explains the negative correlation because no fetus is present, and colostrum production remains for only a few days. Furthermore, the immunoglobulin concentrations in the colostrum decrease even though the demand for $\mathrm{Ca}^{++}$remains high. However, the $\mathrm{Ca}^{++}$concentrations quickly return to normal via regulatory mechanisms (Goff \& Horst, 1997; Santos et al., 2012).

The albumin behavior during this study period was similar to that observed for the total protein and agrees with the results of Sadjadian et al. (2013). However, the antepartum albumin indices were below the normal range (Kaneko et al., 2008), which does not agree with the findings in dairy goats by Balıkc1 et al. (2007) or in sheep by Santos et al. (2012), who found no differences in the concentrations of this variable during that period.

According to Caldeira (2005), the blood albumin concentration is used as an indicator of liver function and nutritional status and is an important labile protein reserve that the animals resort to during times of nutritional deficiency. Therefore, our findings reflect the higher protein demand of dairy goats than of sheep. No correlation was observed between ionized calcium and albumin; however, this protein is the major calcium carrier in the blood stream that directly influences total calcium concentrations (Heras-Herzig \& Guise, 2008).

The progressive increase in serum urea found in this work agrees with the results obtained by Sadjadian et al. (2013), who attributed this behavior to the increase in postpartum dry matter intake. These findings disagree with Piccione et al. (2009) and Santos et al. (2012), who observed no differences in the concentrations of this variable in sheep during the transitional period. The latter authors attribute their findings to appropriate nutritional management because urea responds quickly to changes in dietary protein intake.

This increase in urea values reflects the provision of a diet with high protein and energy demand because the urea present in the bloodstream uses ammonia as its precursor. This may be of rumenal origin or may originate from amino acid (AA) catabolism, nucleic acids and other nitrogenous compounds (Caldeira, 2005). The moderately negative relationship observed between ionized calcium and urea in both groups was due to a progressive increase of the latter, with a concomitant 
reduction in $\mathrm{Ca}^{++}$at the end of the first time point. As has already been noted, this reflects the excess protein and dietary energy and the high calcium demands in this period (Horst et al., 1994; Caldeira, 2005).

Serum creatinine concentrations were below the normal range for the species throughout the study period in both groups (Kaneko et al., 2008). In normoglycemic animals, the highest values are observed at birth, as observed by Santos et al. (2012). Those authors attributed this high value to the muscle protein mobilization used to produce energy during a period of low food intake. Therefore, the moderately positive correlation between ionized calcium and creatinine likely occurred because, while the high demand for $\mathrm{Ca}^{++}$lowered the serum levels, diets with high protein and energy concentrations reduce serum creatinine concentrations. Notably, although creatinine concentrations are largely unaffected by diet or protein catabolism (Russell \& Roussel, 2007), the creatinine concentration does depend on the volume of the muscle mass, which is dependent on diet quantity and quality (Piccione et al., 2009).

CK values near the upper limit or slightly elevated during the postpartum period may result from the intensive management of these animals at this time. This enzyme is a highly sensitive and specific indicator of muscle injury; thus, sudden increases in their activity may occur as a result of increased muscle activity (exercise), mechanical trauma, intramuscular injection or even prolonged recumbency, as in the hypocalcemic animals (Russell \& Roussel, 2007). However, no correlation was observed between the ionic calcium and CK in animals with subclinical hypocalcemia.

The AST serum activity was below the reference values at all time points analyzed, according to Kaneko et al., (2008), but were in accordance with the results of Mundim et al. (2007) and Sadjadian et al. (2013). However, several GGT values were slightly above the normal range for the species (Iriadam, 2007; Mundim et al., 2007; Kaneko et al., 2008).

The total calcium behavior throughout the study periods was in accordance with the results of Azab \& Abdel-Maksoud (1999) and Iriadam (2007). According to those authors, total calcium decreases in late pregnancy and reaches its lowest values at delivery, remaining low during the first 3 weeks after parturition. This can be attributed to the high demand for calcium by the developing fetal skeleton and early milk production. Furthermore, the total calcium is influenced by serum albumin concentrations and, as already discussed, the serum albumin concentrations decline shortly before delivery. At delivery, they negatively influence the total calcium values in this period (Heras-Herzig \& Guise, 2008; Reece, 2017).

No correlation between ionic calcium and total calcium was observed, likely because the latter is more influenced by albumin values, whereas no correlation was observed between albumin and ionic calcium. This was elucidated by Vieira (2007), for whom it was clear that any change in serum protein levels, especially albumin, leads to a change in serum total calcium, which does not indicate a change in the ionized fraction. This explains why, in animals with clinical hypocalcemia, reductions in total serum calcium are not always associated with clinically severe signs, since only the ionized fraction is biologically active.

The phosphorus results for G1 agree with those obtained by Azab and Abdel-Maksoud (1999), who found no difference in serum concentrations of this mineral in goats during the transitional period. The values of this variable were within the normal range for the species in both groups (Kaneko et al., 2008). The uniformity of the values for this mineral in the bloodstream suggests an adequate supply of this element in the diet and an especially efficient phosphorus metabolism (Iriadam, 2007).

Maintenance of phosphorus values that are greater in G1 than in G2 may be related to dietary regulation by active vitamin D metabolites, the parathyroid hormone and calcitonin; therefore, low serum phosphorus activates these regulatory mechanisms (Carlson, 2006). At low values of phosphorus, ruminants increase the affinity of active vitamin D3 (1,25dihydroxicolecalciferol) receptors in the small intestine, which favors calcium absorption, but this response does not increase the serum concentrations of this vitamin (Schröder et al., 1997). Therefore, the higher phosphorus concentrations in G1 may have negatively interfered in vitamin D3 action. However, Oetzel (1988), Bruére and West (1993) and Darrell et al. (2005) 
reported that some cases of clinical hypocalcemia are accompanied by hypophosphatemia, which can be associated with anorexia in these animals; therefore, this finding is not present in animals that are subclinically affected.

Serum magnesium concentrations in both groups, as well as the behavior of magnesium during the study period, were similar to those of Azab and Abdel-Maksoud (1999), who found a reduction in serum levels of this mineral at delivery. According to Kimberling (1988), magnesium interferes with serum calcium concentrations because low plasma levels of this mineral can make the bone tissue refractory to parathyroid hormone action. Therefore, the higher magnesium values in G1 may have possibly contributed to the lack of clinical cases. This, combined with the fact that serum magnesium concentrations are directly related to the absorption of this element, especially in the rumen (Berchielli et al., 2011), may explain the moderately positive correlation observed between $\mathrm{Ca}^{++}$and magnesium in this study.

The contents of sodium, potassium and chloride observed in this study are in agreement with the findings of Azab and Abdel-Maksoud (1999) and Piccione et al. (2009), who found that the plasma concentrations of these elements were within the normal range for goats during the transitional period and in nonpregnant and nonlactating goats, respectively, as described by Kaneko et al. (2008).

\section{Conclusion}

In conclusion, considerable interference from $\mathrm{Ca}^{++}$occurs in the metabolism of dairy goats during the transitional period, particularly with regard to energy metabolism and insulin. The cutoff point defined for $\mathrm{Ca}^{++}\left(\mathrm{Ca}^{++}<0.73\right)$ was correct because there were significant differences in the behavior of biochemical indicators and the subclinical occurrence of pregnancy toxemia between the group of goats with subclinical hypocalcemia and normocalcemia. Therefore, goats with subclinical hypocalcemia have a higher risk of developing other diseases in this period and lower production rates than normocalcemic goats, as is also true for cows. Subclinical hypocalcemia occurs in dairy goats particularly at parturition and during the first 10 days postpartum, as well as at peak production, which corresponds to 40 to 50 days postpartum. The defined cutoff point in this pioneering work is adequate and recommended for the diagnosis of subclinical hypocalcemia in dairy goats.

That is a lack of information regarding the occurrence and consequences of subclinical hypocalcemia and its importance in small ruminants, especially dairy goats. Therefore, researches that aim to quantify the economic losses caused by subclinical hypocalcemia, its correlation with other diseases such as: pregnancy toxemia, hypomagnesemia, mastitis , placental retention, metritis, endometritis and pneumonia, as well as the particularities of calcium control and metabolism in goats and sheep should be developed.

\section{Acknowledgments}

We are grateful to the Conselho Nacional de Desenvolvimento Científico e Tecnológico (CNPq, Project 4760502012-0) for the financial support and the MSc grant obtained by Mr. Jobson Filipe de Paula Cajueiro.

\section{References}

Araújo, D. F. I. \& Silva, P. (2008). Valores de amilase, colesterol e triglicérides em soro de cabras de Mossoró, RN. Acta Veterinaria Brasilica, 3(3), 97-100. https://doi.org/10.21708/avb.2008.2.3.903

Azab, M. E. \& Abdel-Maksoud, H. A. (1999). Changes in some hematological and biochemical parameters during prepartum and postpartum periods in female Baladi goats. Small Ruminant Research, 34(1), 77-85. https://doi.org/10.1016/S0921-4488(99)00049-8

Balıkcı, E., Yıldız, A. \& Gürdoğan, F. (2007). Blood metabolite concentrations during pregnancy and postpartum in Akkaraman ewes. Small Ruminant Research, 67(2), 247-251. 10.1016/ j.smallrumres.2005.10.011

Barbosa, L. P., Rodrigues, M. T., Guimarães, J. D., Maffili, V. V., Amorim, L. S. \& Garcez Neto, A. F. (2009). Condição corporal ao parto e perfil metabólico de cabras alpinas no início da lactação. Revista Brasileira de Zootecnia, 38(10), 2007-2014. https://doi.org/10.1590/S1516-35982009001000022 
Basoglu, A., Sevinç, M., Mahmut, O. K. \& Gökçen, M. (1998). Peri and postparturient concentrations of lipid lipoprotein insulin and glucose in normal dairy cows. Turkish Journal of Veterinary and Animal Sciences, 22, 141-144.

Bell, A. W. \& Bauman, D. E. (1997). Adaptations of glucose metabolism during pregnancy and lactation. Journal Mammary Gland Biology Neoplasia, 2(3), 265-278. 10.1023/A:1026336505343.

Bell, A. W. (1995). Regulation of organic nutrient metabolism during transition from late pregnancy to early lactation. Journal of Animal Science, 73(9), 28042819. 10.2527 / 1995.7392804x.

Bennis, A., De La Farge, F., Bézille, P., Valdiguié, P., Rico, A. G. \& Braun, J. P. (1992). Effects of age of newborn and delivery by female goats on plasma lipids and lipoproteins. Small Ruminant Research, 9(3), 243-253. https://doi.org/10.1016/0921-4488(92)90154-V

Berchielli, T. T., Pires, A. V., Oliveira, S. G. (2011). Nutrição de Ruminantes. (2th ed.), FAPPESP/FUNESP, 615p.

Brozos, C., Mavrogianni, V. S. \& Fthenakis, G. C. (2011). Treatment and control of peri-parturient metabolic diseases: Pregnancy toxemia, hypocalcemia, hypomagnesemia. Veterinary Clinics of North America: Food Animal Practice, 27(1), 105-113. 10.1016/ j.cvfa.2010.10.004.

Bruére, A. N. \& West, D. M. (1993). The sheep: Health, disease \& profuction. Veterinary Continuing Education: Palmerston North, New Zealend. 408p.

Caldeira, R. M. \& Portugal, A. V. (1991). Interrelationship between body condition and metabolic status in ewes. Small Ruminant Research, 6, 15-24. https://doi.org/10.1016/0921-4488(91)90003-9

Caldeira, R. M. (2005). Monitorização da adequação do Plano alimentar e do estado nutricional em ovelhas. Revista Portuguesa de Ciências Veterinárias, 100, 125-139.

Campos, R., Hernández, E. A., Giraldo, L. \& González, F. (2009). Cortisol e sua relação com a regulação endócrina no período de transição em vacas leiteiras sob condições do trópico colombiano. Ciência Animal Brasileira, 1, 790-794.

Carlson, G. P. (2006). Testes bioquímicos. In: Smith, B. P., De Souza Coutinho, A., Lopes Leite, C. A., Figueiredo, C., Ames, T. R. \& Aleman, M. R. Medicina interna de grandes animais. (3th ed.), 389-412.

Constable, P. D., Hinchcliff, K. W., Done, S. H. \& Grunberg, W. (2017). Veterinary Medicine: A Textbook of the Diseases of Cattle, Horses, Sheep, Pigs, and Goats. 11th ed. Elsevier, St. Louis, MO, USA, 2278p.

Curi, P. R. (1997). Metodologia e Análise da Pesquisa em Ciências Biológicas. Tipomic, 263p.

Darrell, L. R, Jr., Debra, C. R. \& Pugh, D. G. (2005). Alimentação e nutrição. In: Pugh, D. G., Fagliari, J. J. \& Anderson, D. E. Clínica de ovinos e caprinos. Roca, 52-53.

De Koster, J. D \& Opsomer, G. (2013). Insulin resistance in dairy cows. Veterinary Clinics of North America: Food Animal Practice, 29(2), 299-322. 10.1016 / j.cvfa.2013.04.002

DeGaris, P. J. \& Lean, I. J. (2008). Milk fever in dairy cows: A review of pathophysiology and control principles. The Veterinary Journal, 176(2009), 58-69. 10.1016/j.tvj1.2007.12.029

Duehlmeier, R., Fluegge, I., Schwert, B. \& Ganter, M. (2013). Insulin sensitivity during late gestation in ewes affected by pregnancy toxemia and in ewes with high and low susceptibility to this disorder. Journal of Veterinary Internal Medicine, 27(2), 359-366. 10.1111/ jvim.12035

Reece, W. O. (2017). Dukes - Fisiologia dos animais domésticos. (13th ed.), Roca. 740p.

El-Belely, M. S., Al-Qarawi, A. A. \& Abdel-Rahman, H. A. J. (2000). Interrelationships between the blood coagulation profile and plasma concentrations of progesterone, oestradiol-17 $\beta$ and cortisol throughout pregnancy and around parturition in sheep. The Journal of Agricultural Science, 135(2), 203-209. https://doi.org/10.1017/S0021859699008114

Goff, J. P. \& Horst, R. L. (1997). Physiological changes at parturition and their relationship to metabolic disorders. Journal Dairy Science, 80(7), 1260-1268. 10.3168 / jds.S0022-0302 (97) 76055-7.

Goff, J. P. (2000). Pathophysiology of calcium and phosphorus disorders. Veterinary Clinics of North America: Food Animal Practice, 16(2), 319-337. 10.1016 / s0749-0720 (15) 30108-0.

Goff, J. P. (2006). Major advances in our understanding of nutritional influences on bovine health. Journal Dairy Science, 89(4), 1292-1301. 10.3168 / jds.S0022-0302 (06) 72197-X.

Goff, J. P. (2008). The monitoring, prevention, and treatment of milk fever and subclinical hypocalcemia in dairy cows. Veterinary jornal, 176(1), 50-57. $10.1016 /$ j.tvj1.2007.12.020.

González, F. H. D., Silva, S. C. (2017). Introdução à bioquímica clínica veterinária. UFRGS. 535p.

González, F. H. D. \& Scheffer, J. F. S. (2002). Perfil sanguíneo: Ferramenta de análise clínica, metabólica e nutricional. Congresso Naciona de Medicina Veterinária, 29, 5-17.

Grummer, R. R. (1995). Impact of changes in organic nutrient metabolism on feeding the transition dairy cow. Journal of Animal Science, 73(9), 2820-2833. $10.2527 / 1995.7392820 x$.

Gupta, V. K., Kumar, A. \& Vihan, V. S. (2005). Clínical parturient paresis (milk fever) in goat - A case report. Research Journal for Veterinary Practitioners, 6(1), 17. 
Heras-Herzig, A. \& Guise, T. A. (2008). Disorders of calcium metabolism. In: Alpern, R. J., Herbert, S. C., Seldin, D. W. \& Giebisch G. H. Seldin and Giebisch's the kidney: Physiology and pathophysiology. 4th ed. Elsevier Academic Press, Amsterdam, Netherlands. 1911-1944.

Horst, R. L. \& Jorgensen, N. A. (1982). Elevated plasma cortisol during induced and spontaneous hypocalcemia in ruminants. Journal Dairy Science, 65(12), 2332-2337. 10.3168 / jds.S0022-0302 (82) 82505-8.

Horst, R. L., Goff, J. P. \& Reinhardt, T. A. (2005). Adapting to the transition between gestation and lactation: Differences between rat, human and dairy cow. Journal Mammary Gland Biology Neoplasia, 10(2), 141-156. 10.1007 / s10911-005-5397-x.

Horst, R. L., Goff, J. P. \& Reinhardt, T. A. (1994). Calcium and vitamin D metabolism in the dairy Cow. Journal Dairy Science, 77(7), 1936-1951. 10.3168 / jds.S0022-0302 (94) 77140-X.

Huzzey, J. M., Nydam, D. V., Grant, R. J. \& Overton, T. R. (2011). Associations of prepartum plasma cortisol, haptoglobin, fecal cortisol metabolites, and nonesterified fatty acids with postpartum health status in Holstein dairy cows. Journal Dairy Science, 94(12), 5878-5889. 10.3168 / jds.2010-3391.

Iriadam, M. (2007). Variation in certain hematological and biochemical parameters during the peri-partum period in Kilis does. Small Ruminant Research, 73(1), 54-57. 10.1016/ j.smallrumres.2006.11.001

Kaneko, J. J., Harvey, L. W. \& Bruss, M. L. (2008). Clinical biochemistry of domestic animals. (6th ed.) Academic Press, 936p.

Kimberling, C. V. (1988). Diseases of ewes. In: Jensen, R., Swift, B. L. \& Kimberling, C. V. Jensen and Swift's diseases of sheep. 3th ed. Lea \& Febiger, Philadelphia, PA. 26-29.

Kimura, K., Reinhardt, T. A. \& Goff, J. P. (2006). Parturition and hypocalcemia blunts calcium signals in immune cells of dairy cattle. Journal Dairy Science, 89(7), 2588-2595. 10.3168 / jds.S0022-0302 (06) 72335-9

Liesegang, A. \& Risteli, J. (2005). Influence of different calcium concentrations in the diet on bone metabolism in growing dairy goats and sheep. Journal of animal physiology and animal nutrition, 89(3-6), 113-119. 10.1111/j.1439-0396.2005.00548.x

Lima, E. H. F., Mendonça, C. L., Cajueiro, J. F. P., Carvalho, C. C. D., Soares, P. C., Souto, R. J. C., Drummond, A. R. F. \& Afonso, J. A. B. (2016). Efeito da monensina sódica sobre o perfil metabólico de ovelhas antes e após o parto. Ciência Animal Brasileira, 17(1), 105-118. https://doi.org/10.1590/10896891v17i128370

McGuire, M. A., Griinari, J. M., Dwyer, D. A. \& Bauman, D. E. (1995). Role of insulin in the regulation of mammary synthesis of fat and protein. Journal Dairy Science, 78(4), 816-824. 10.3168 / jds.S0022-0302 (95) 76693-0.

Moreno-Rojas, R., Zurera-Cosano, G. \& Amaro-Lopez, M. A. (1994). Concentration and seasonal variation of calcium, magnesium, sodium and potassium in raw cow, ewe and goat milk. International Journal of Food Sciences and Nutrition, 45, 99-105. doi.org/10.3109/09637489409166148

Mundim, A. V., Costa, A. S., Mundim, S. A. P., Guimarães, E. C. \& Espindola, F. S. (2007). Influência da ordem e estádios da lactação no perfil bioquímico sangüíneo de cabras da raça Saanen. Arquivo Brasileiro de Medicina Veterinária e Zootecnia, 59(2), 306-312.

Oetzel, G. R. (1988). Parturient paresis and hypocalcemia in ruminant livestock. Veterinary Clinics of North America: Food Animal Practice, 4(2), 351-364. doi.org/10.1016/S0749-0720(15)31053-7

Ortolani, E. L. (1995). Aspectos clínicos, epidemiologicos e terapeuticos da hipocalcemia de vacas leiteiras. Arquivo Brasileiro de Medicina Veterinária e Zootecnia 47(6), 799-808

Piccione, G., Caola, G., Giannetto, C., Grasso, F., Runzo, S. C., Zumbo, A. \& Pennisi, P. (2009). Selected biochemical serum parameters in ewes during pregnancy, post-parturition, lactation and dry period. Animal Science Papers and Reports, 27(4), 321-330.

Rabelo, E., Rezende, R. L., Bertics, S. J. \& Grummer, R. R. (2005). Effects of pre- and postfresh transition diets varying in dietary energy density on metabolic status of periparturient dairy cows. Journal Dairy Science, 88(12), 4375-4383. doi.org/10.3168/jds.S0022-0302(05)73124-6

Reinhardt, T. A., Lippolis, J. D., McCluskey, B. J., Goff, J. P. \& Horst, R. L. (2011). Prevalence of subclinical hypocalcemia in dairy herds. Veterinary journal,188(1), 122-124. 10.1016/ j.tvj1.2010.03.025

Ríos, C., Marín, M. P., Catafau, M. \& Wittwer, F. (2006). Concentraciones sanguíneas de B-hidroxibutirato, NEFA, colesterol y urea en cabras lecheras de tres rebaños con sistemas intensivos de producción y su relación con el balance nutricional. Archivos de Medicina Veterinária, 38(1), 19-23. dx.doi.org/10.4067/S0301-732X2006000100003.

Russell, K. E. \& Roussel, A. J. (2007). Evaluation of the ruminant sérum chemistry profile. Veterinary Clinics of North America: Food Animal Practice, 23(3), 403-426. 10.1016/ j.cvfa.2007.07.003.

Sadjadian, R., Seifi, H. A., Mohri, M., Naserian, A. A. \&. Farzaneh, N. (2013). Variations of energy biochemical metabolites in periparturient dairy Saanen goats. Comparative Clinical Pathology, 22(3), 449-456. 10.1007 / s00580-012-1431-8

Santos, J. E. P. (2011). Distúrbios metabólicos. In: Pedroso, A. M., Pires, A. V., Garcia, A. V. \& Ribeiro, C. V. D. M., Nutrição de Ruminantes, (2th ed.) Funep, Jaboticabal, SP. 439-520.

Santos, R. A., Campos, A. G. S. S., Afonso, J. A. B., Soares, P. C. \& Mendonça, C. L. (2012). Efeito da administração de propileno glicol e cobalto associado à vitamin $\mathrm{A}_{12}$ sobre o perfil metabólico e a atividade enzimática de ovelhas da raça Santa Inês no Periparto. Pesquisa Veterinária Brasileira, $32: 60-66$.

Schlumbohm, C. \& Harmeyer, J. (2003). Hypocalcemia reduces endogenous glucose production in hyperketonemic sheep. Journal Dairy Science, 86(6), 1953-1962. doi.org/10.3168/jds.S0022-0302(03)73783-7 
Schlumbohm, C., Sporleder, H. P., Gürtler, H. \& Harmeyer, J. (1997). The influence of insulin on metabolism of glucose, free fatty acids and glycerol in normo and hipocalcêmic ewes during different reproductive states. Deutsche tierärztliche Wochenschrift, 104, 359-365.

Schlumbohm, C. \& Harmeyer, J. (1990). Hypocalcemia reduces rate of disappearance of glucose from plasma. Zentralblatt fur Veterinarmedizin. Reihe A, 37(4), 285-293. 10.1111/ j.1439-0442.1990.tb00907.x

Schröder, B. \& Breves, G. (2006). Mechanisms and regulation of calcium absorption from the gastrointestinal tract in pigs and ruminants: comparative aspects with special emphasis on hypocalcemia in dairy cows. Animal health research reviews, 7(1-2), 31-41. 10.1017 / S1466252307001144.

Schröder, B., Rittmann, I., Pfeffer, E. \& Breves, G. (1997). In vitro studies on calcium absorption from the gastrointestinal tract in small ruminants. Journal of Comparative Physiology, 167, 43-51. 10.1007/s003600050046.

Simplício, K., Cotrim, F., Fagliari, J. J. \& Nagib, R. L. J. (2009). Perfil bioquímico sérico de cabras da raça saanem e Boer. Congresso Braseiro de Buiatria, 8 , 270-273.

Smith, M. C. \& Sherman, D. M. (2009). Nutrition and metabolic diseases. In: Smith, M. C. \& Sherman, D. M. Goat medicine. (2th ed.), Wiley-Blackwell, Ames, IA. 761-763.

Soares, G.S.L., Souto, R. J. C., Cajueiro, J.F.P., Afonso, J. A. B., Rego, R.O., Macêdo, A.T.M., Soares, P. C. \& Mendonça, C. L. (2018). Adaptive changes in blood biochemical profile of dairy goats during the period of transition. Revue Médicine Vétérinarie, 169(1-3), 65-75.

Souto, R. J. C., Afonso, J. A. B., Mendonça, C. L., Carvalho, C. C. D., Silva Filho, A. P., Cajueiro, J. F. P., Lima, E. H. F. \& Soares, P. C. (2013). Achados bioquímicos, eletrolíticos e hormonais de cabras acometidas com toxemia da prenhez. Pesquisa Veterinária Brasileira, 33(10), 1174-1182.

Thrusfield, M., Christley, R., Brown, E., Diggle, P. J., French, N., Howe, K., Kelly, L., O'Connor, A., Sargeant, j. \& Wood, H. (2018). Veterinary Epidemiology. (4th ed.), Wiley-Blackwell, Hoboken, N.J. 319-338.

Van der Drift, S. G., Houweling, M., Schonewille, J. T., Tielens, A. G. \& Jorritsma, R. (2012). Protein and fat mobilization and associations with serum $\beta$ Hydroxybutyrate concentrations in dairy cows. Journal Dairy Science, 95(9), 4911-4920. doi.org/10.3168/jds.2011-4771

Vieira, J. G. H. (2007). Diagnostico laboratorial e monitoramento das doenças osteometabolicas. Jornal Brasileiro de Patologia e Medicina Laboratorial, 43(2), 75-82.

Walz, H. A., Wierup, N., Vikman, J., Manganiello, V. C., Degerman, E., Eliasson, L. \& Holst, L. S. (2007). $\beta$-cell PDE3B regulates Ca ${ }^{++}$stimulated exocytosis of insulin. Cellular signalling, 19(7), 1505-1513. 10.1016 / j.cellsig.2007.01.030.

Zambom, M. A., Alcalde, C. R., Macedo, F. A. F., Garcia, J., Moraes, G. V., Sakuno, M. L. D. \& Borgh, E. L. (2006). Ingestão digestibilidade das rações e parâmetros sangüíneos em cabras Saanen durante o pré-parto recebendo rações com diferentes níveis de energia. Revista Brasileira de Zootecnia, 35(4), 18661871. 10.1590 / S1516-35982006000600039 